\title{
Cardiac CT angiography: normal and pathological anatomical features-a narrative review
}

\author{
Alberto Clemente ${ }^{1}$, Sara Seitun ${ }^{2}$, Cesare Mantini ${ }^{3}$, Giovanni Gentile ${ }^{4}$, Duccio Federici ${ }^{5}$, Andrea Barison ${ }^{6}$, \\ Andrea Rossi ${ }^{7}$, Magdalena Cuman ${ }^{8}$, Alessandra Pizzuto ${ }^{8}$, Lamia Ait-Ali ${ }^{9}$, Eduardo Bossone ${ }^{10}$, \\ Filippo Cademartiri ${ }^{11}$, Dante Chiappino ${ }^{1}$
}

${ }^{1}$ Department of Radiology, CNR (National Council of Research)/Tuscany Region "Gabriele Monasterio" Foundation (FTGM), Massa, Italy; ${ }^{2}$ IRCCS Policlinico San Martino Hospital, Genoa, Italy; ${ }^{3}$ Department of Neuroscience, Imaging and Clinical Science, Institute of Radiology, "G. d'Annunzio" University, Chieti, Italy; ${ }^{4}$ Radiology Unit, IRCCS ISMETT (Mediterranean Institute for Transplantation and Advanced Specialized Therapies), Palermo, Italy; ${ }^{5}$ Pediatric Cardiac Surgery, CNR (National Council of Research)/Tuscany Region "Gabriele Monasterio" Foundation (FTGM), Massa, Italy; ${ }^{6}$ Cardiology Division, CNR (National Council of Research)/Tuscany Region "Gabriele Monasterio" Foundation (FTGM), Pisa, Italy; ${ }^{7}$ Arrhythmology Unit, Department of Invasive Cardiology, CNR (National Council of Research)/Tuscany Region "Gabriele Monasterio" Foundation (FTGM), Pisa, Italy; ${ }^{8}$ Pediatric Cardiology and GUCH Unit, CNR (National Council of Research)/Tuscany Region "Gabriele Monasterio" Foundation (FTGM), Massa, Italy; ${ }^{9}$ Institute of Clinical Physiology, National Research Council, Pisa, Italy; ${ }^{10}$ Department of Cardiology, Ospedale Cardarelli, Naples, Italy; ${ }^{11}$ SDN IRCCS, Naples, Italy

Contributions: (I) Conception and design: A Clemente, F Cademartiri; (II) Administrative support: A Clemente, S Seitun, F Cademartiri; (III) Provision of study materials or patients: A Clemente, S Seitun, C Mantini, G Gentile; (IV) Collection and assembly of data: All authors; (V) Data analysis and interpretation: All authors; (VI) Manuscript writing: All authors; (VII) Final approval of manuscript: All authors.

Correspondence to: Sara Seitun, MD. Department of Radiology 1, IRCCS Policlinico San Martino Hospital, Genoa, Italy. Email: saraseitun@yahoo.com.

\begin{abstract}
The normal and pathological anatomy of the heart and coronary arteries are nowadays widely developed topics and constitute a fundamental part of the cultural background of the radiologist. The introduction of cardiac ECG-gated synchronized CT scanners with an ever-increasing number of detectors and with increasingly high structural characteristics (increase in temporal resolution, increase in contrast resolution with dual-source, dual energy scanners) allows the virtual measurement of anatomical in vivo structures complying with heart rate with submillimetric precision permitting to clearly depict the normal anatomy and follow the pathologic temporal evolution. Accordingly to these considerations, cardiac computed tomography angiography (CCTA) asserts itself as a gold standard method for the anatomical evaluation of the heart and permits to evaluate, verify, measure and characterize structural pathological alterations of both congenital and acquired degenerative diseases. Accordingly, CCTA is increasingly used as a prognostic model capable of modifying the outcome of diseased patients in planning interventions and in the post-surgical/interventional follow-up. The profound knowledge of cardiac anatomy and function through highly detailed CCTA analysis is required to perform an efficient and optimal use in real-world clinical practice.
\end{abstract}

Keywords: Cardiac computed tomography angiography (CCTA); cardiac anatomy; normal heart anatomy; cardiac chambers; cardiac functional parameters; cardiac valves; heart valve diseases; coronary artery disease (CAD); coronary artery anomalies; congenital; heart defects; cardiology interventions; cardiac catheterization

Submitted May 24, 2020. Accepted for publication Dec 07, 2020.

doi: $10.21037 / \mathrm{cdt}-20-530$

View this article at: http://dx.doi.org/10.21037/cdt-20-530 


\section{Introduction}

The knowledge of normal anatomy has always aroused, in past centuries, the interest of many scholars and nowadays plays a fundamental role in the training of imaging specialists and in the recognition of cardiac disease.

In the past, with the birth of an anthropocentric culture in the Renaissance, the use of the experimental approach to the nature of the "homo faber" and the establishment of the aesthetic canon of the "measure" as a synthesis of nature and representation of life, the anatomy was studied in sequential layers (Alessandro Allori). Leonardo as "homo sanza lettere" increased his anatomical studies over the classical texts, verifying in practice the hypotheses of his contemporaries. In this way drawn anatomy was born, marking the transition from the static surface anatomy, to the depth anatomy, dynamic by nature, of the dissected, passing from an artistic anatomy to a medical anatomy (1). As for Leonardo, anatomy is a point of convergence of statics and dynamics. Today these concepts are in vivo evaluated by the non-invasive study of cardiac structures through cardiac computed tomography angiography (CCTA). As Leonardo initially passes from the analysis of axial images (horizontal anatomy) to the representation of the heart seen from the inside or analytically sectioned to understand the relationships between the parts, today this is possible through CCTA that creates volumetric data capable of providing information of cardiac morphology, function and vitality. Furthermore, the interpretation of cardiac structure, function (using mathematical models of image reconstructions) and multidimensional algorithms that permit a dynamic evaluation, allows not only a qualitative but also a quantitative CT evaluation (such as iatrophysical model) of normal and pathologic cardiac structures. Giovanni Battista Morgagni in the second half of the eighteenth century correlated clinical signs and symptoms observed in living patients with morphological substrates found at autopsy and collected his studies in "de sedibus et causis morborum per anatomen indagatis" by which he systematically developed the method of anatomical and clinical correlations. He collected pathologic cases and in this way, he was able to identify many concealed aspects of the clinical and anatomical features of several morbid entities, contributing to the development of diagnosis with therapeutic implications (2). This anatomo-medical approach has been developed more recently with the passage from analogical radiology to digital advanced cardiac radiology and the use of CCTA provides a detailed volumetric anatomoclinical in-vivo diagnostic value significantly contributing in modifying the prognosis.

The purpose of this review is to describe the state of the art of CCTA anatomic evaluation related to clinical applications, looking at its strengths and limitations.

We present the following article in accordance with the Narrative Review reporting checklist (available at http:// dx.doi.org/10.21037/cdt-20-530).

\section{Cardiac structures}

The heart is a predominantly muscular pump that moves blood into the vessels through the pulmonary and systemic circulation ("vis a tergo"). The four chambers are combined in atrioventricular pairs connected together by valve systems (Figure 1).

The pulmonary and systemic circles are interposed between the two atrioventricular systems (right and left). Systemic venous blood reaches the heart through the upper and lower hollow veins of the right atrium and from here the blood reaches the right ventricle (RV) through the tricuspid valve. During systole, blood is pushed through the pulmonary valve into the pulmonary artery which leads it to the alveolar circle. The oxygenated blood then returns to the heart from the alveolar circle through the multiple pulmonary veins (normally 4 but varying from 3 to 5 ) that flow into the left atrium and from there, through the mitral valve (MV), the blood reaches the left ventricle. During systole, the blood is pushed back through the aortic valve into the systemic circulation and to the coronary arteries responsible for cardiac vascularization (Figure 2, Table 1). These dynamic morpho-functional conditions must be taken into account when planning the CCT investigation for the best optimization of the scan execution.

\section{Left ventricle}

The widespread availability of CT scanners has led to increasing use of CCTA for the noninvasive evaluation of coronary artery disease (CAD). Although CCTA is not recommended as the first-line modality for suspected myocardial aspects, CCTA allows the assessment of ventricular sizes, morphology, function, density, first pass perfusion defects and delayed-enhancement pattern in multiplanar reconstructions with high spatial resolution in short acquisition times. Therefore, CCTA is considered complementary to echocardiography and cardiac magnetic 

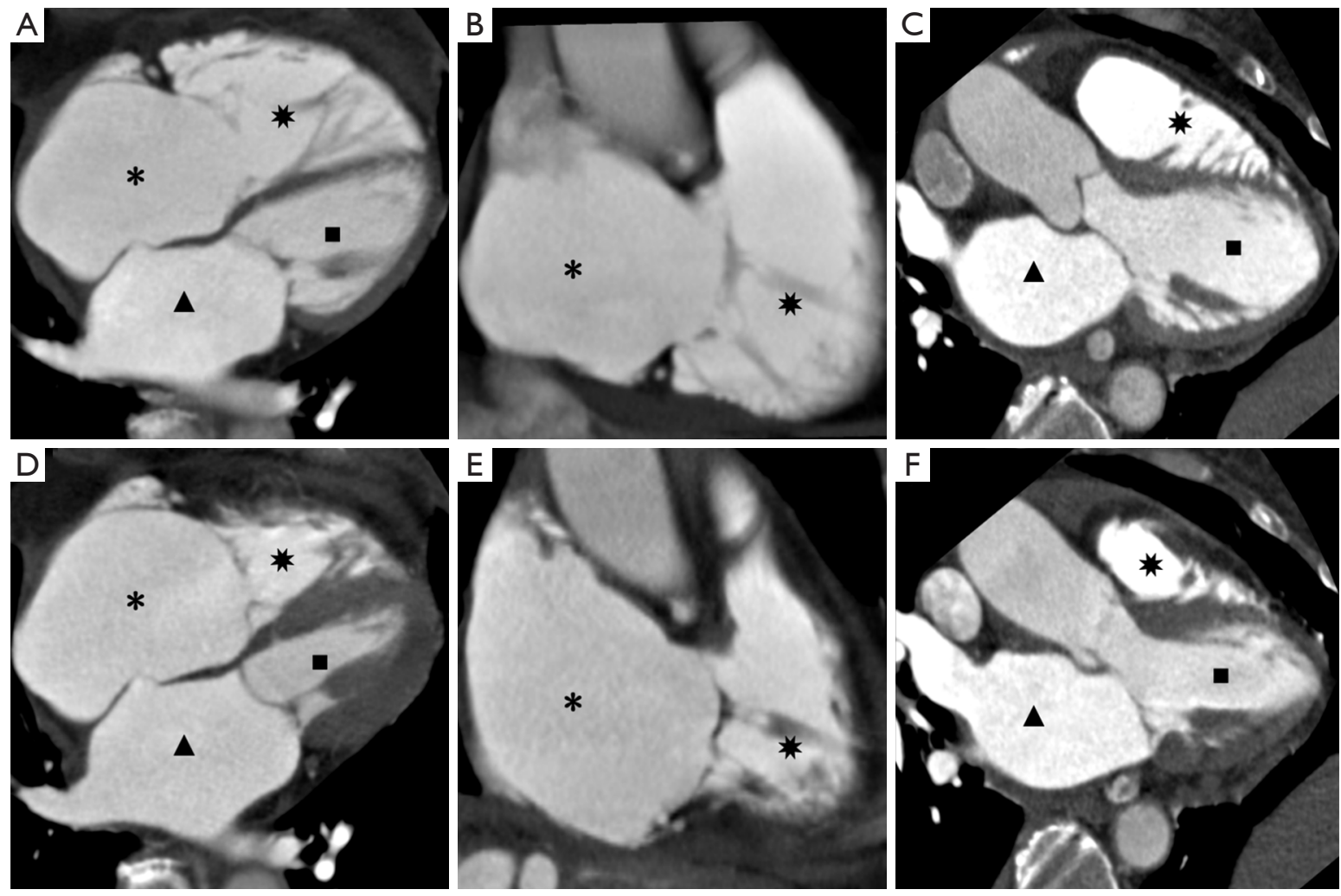

Figure 1 CCTA cardiac planes of chambers visualization: four-chamber view during diastole (A) and systole (D); two-chamber long axis view of the right heart during diastole $(\mathrm{B})$ and systole $(\mathrm{E})$ and two-chamber long axis view of the left heart during diastole (C) and systole (F). *, right atrium; $\boldsymbol{\Delta}$, left atrium; * , right ventricle; $\mathbf{-}$, left ventricle. CCTA, cardiac computed tomography angiography.
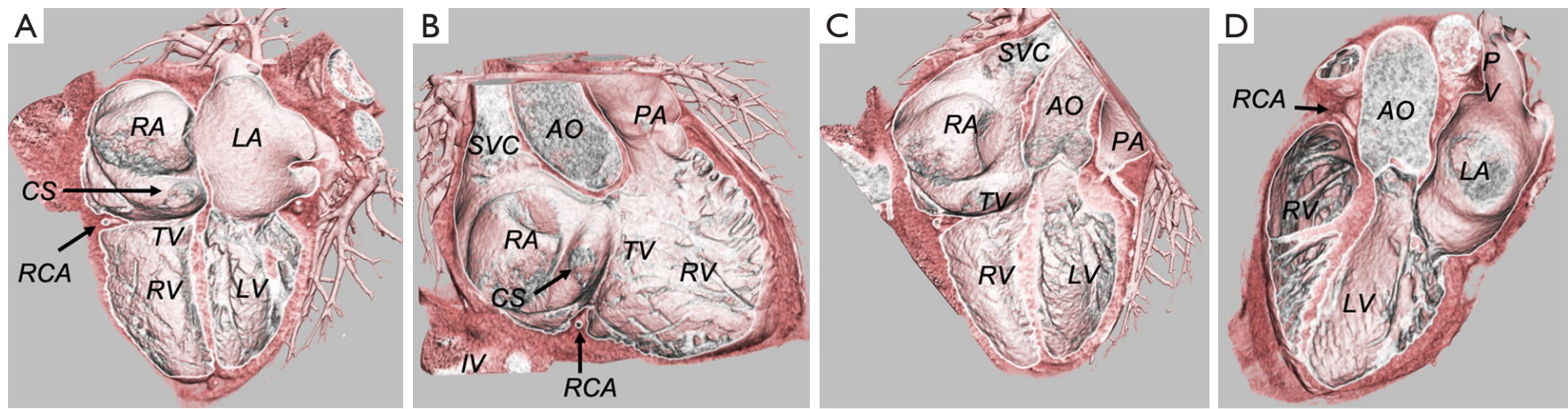

Figure 2 3D-cut plane endoscopy CT images showing the main cardiac chambers and structures. AO, aorta; LA, left atrium; LV, left ventricle; PA, pulmonary artery; PV, pulmonary vein; RCA, right coronary artery; RA, right atrium; RV, right ventricle; RVOT, right ventricular outflow tract; SVC, superior vena cava; IVC, inferior vena cava; TV, tricuspid valve; CS, coronary sinus. 
Table 1 Cardiac structures characteristics

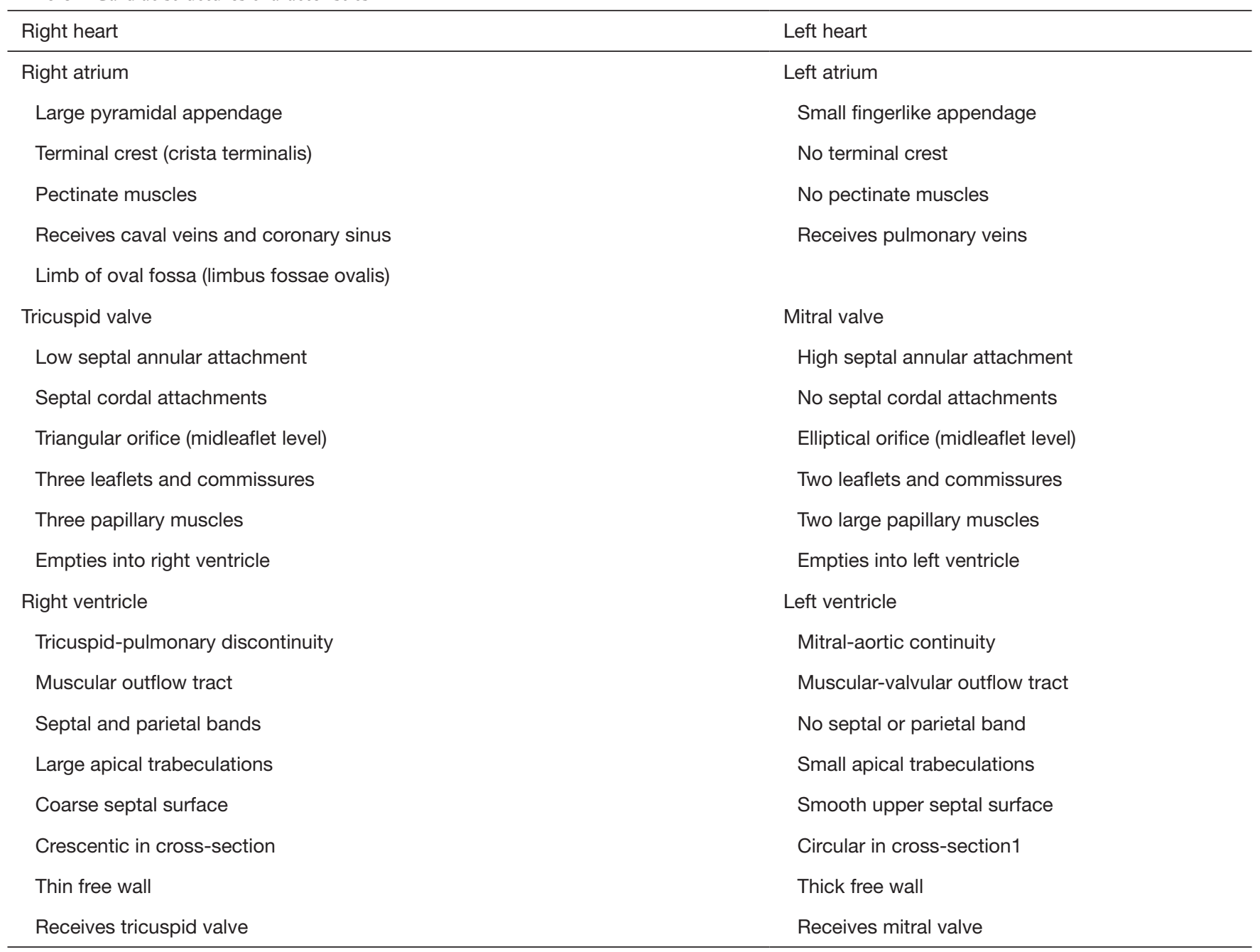

resonance (CMR) in the diagnosis of $\mathrm{LV}$ myocardial diseases in a single sitting $(3,4)$ (Table 2).

With the retrospective ECG-gated spiral scanning, CCTA images are obtained at multiple phases of the cardiac cycle for the assessment of left ventricular (LV) functional parameters including end-diastolic wall thickness, volume, mass and identification of regional wall motion abnormality, approaching the accuracy of CMR (5) (Figure 3). It is also concordant with, and potentially superior to, twodimensional echocardiography, with greater accuracy and reproducibility (6).

CCTA reference standards gender-specific values of LV dimension, volume, mass, and functional parameters (7-13) cannot be extrapolated from echocardiography or CMR due to differences in spatial and temporal resolution and signalto-noise levels. These CT reference values are important for disease classification, risk stratification, and guidance of therapy (3) (Table 3).

Current multidetector row ( $\geq 64$ slices) CT systems allow a reliable and accurate assessment of both coronary epicardial stenosis and its physiological consequences through myocardial rest perfusion during the same acquisition (Figure 4) and additionally stress CT perfusion (CTP) can be performed, making CT the unique noninvasive "one-stop-shop" method. The CTP protocol includes evaluation of myocardial perfusion during both rest (assessment of resting perfusion and coronary arteries) and stress (hyperemia) conditions under coronary vasodilation (e.g., dipyridamole, adenosine). CTP analysis is performed after administration of iodinated contrast medium $(5 \mathrm{~mL} / \mathrm{s})$ which attenuates $\mathrm{X}$-rays directly proportionally to iodine content in tissue; thus myocardial perfusion 
Table 2 Technical characteristics of different imaging modalities

\begin{tabular}{lcccc}
\hline Imaging modality & Spatial resolution & Dimensionality & Temporal resolution & Contrast resolution \\
\hline Catheter angiography & $0.15 \mathrm{~mm}$ & $2 \mathrm{D}$ & $1-10 \mathrm{~ms}$ & Moderate \\
CCTA & $0.23-0.5 \mathrm{~mm}$ & $3 \mathrm{D}$ & $29-175 \mathrm{~ms}$ & Moderate/high* \\
Echocardiography & $0.5-2 \mathrm{~mm}$ & $2 \mathrm{D} / 3 \mathrm{D}$ & $<10 \mathrm{~ms}$ & Low \\
CMR & $1-2 \mathrm{~mm}$ & $2 \mathrm{D} / 3 \mathrm{D}$ & $20-50 \mathrm{~ms}$ & High \\
PET & $4-8 \mathrm{~mm}$ & $3 \mathrm{D}$ & $100-300 \mathrm{~ms}$ & High \\
SPECT & $5-15 \mathrm{~mm}$ & $3 \mathrm{D}$ & $100-300 \mathrm{~ms}$ & High \\
\hline
\end{tabular}

*, high contrast resolution for vascular structures and moderate contrast resolution for myocardial characterization. CCTA, cardiac computed tomography angiography; CMR, cardiac magnetic resonance; SPECT, single-photon emission computed tomography; PET, positron emission tomography.
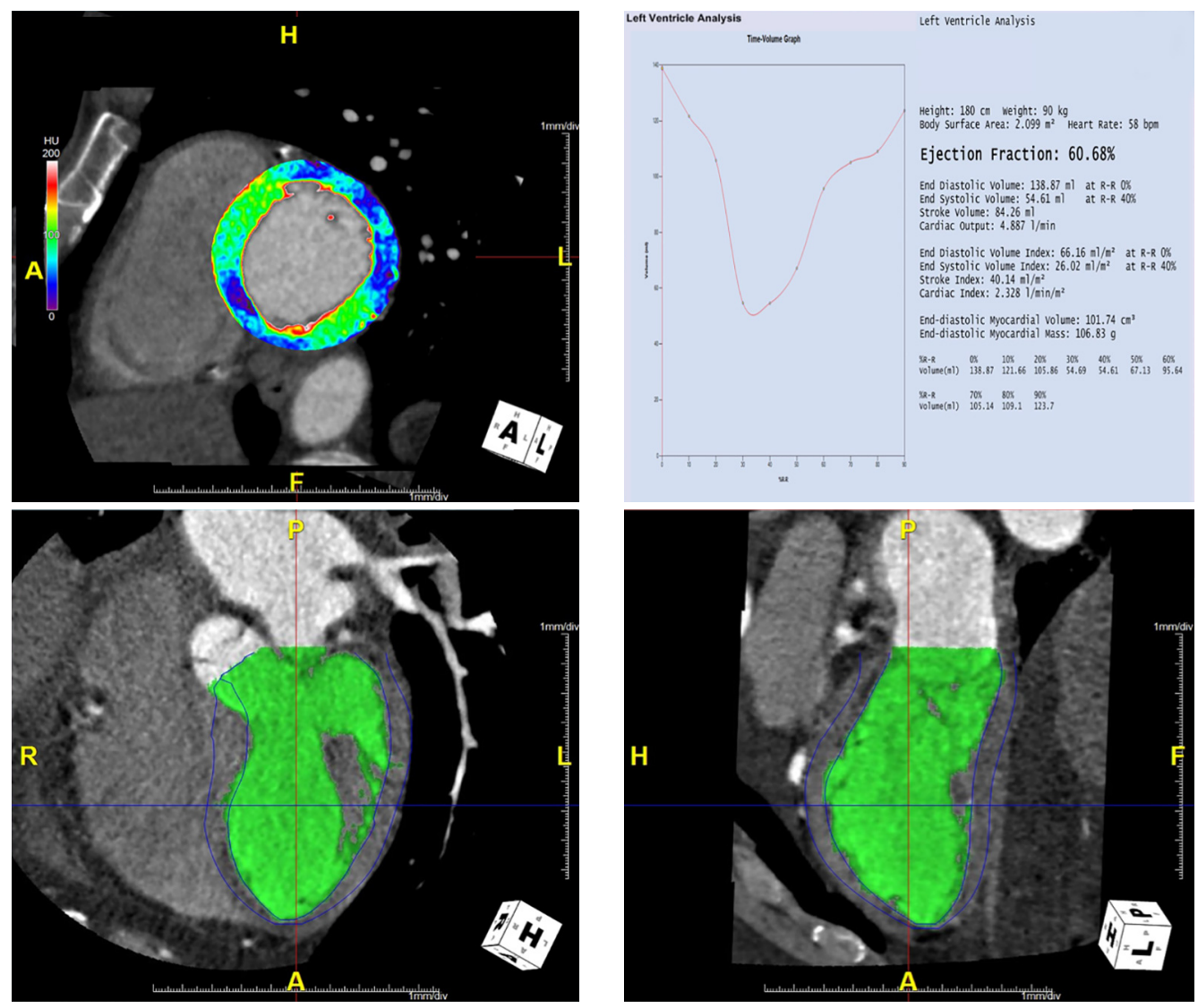

Figure 3 Left ventricle (LV) functional parameters evaluated through CCTA evaluation of both end-diastolic and end-systolic phases. Normal and indexed LV data can be obtained together with global and segmental kinetics of LV and myocardial rest perfusion abnormalities. CCTA, cardiac computed tomography angiography. 


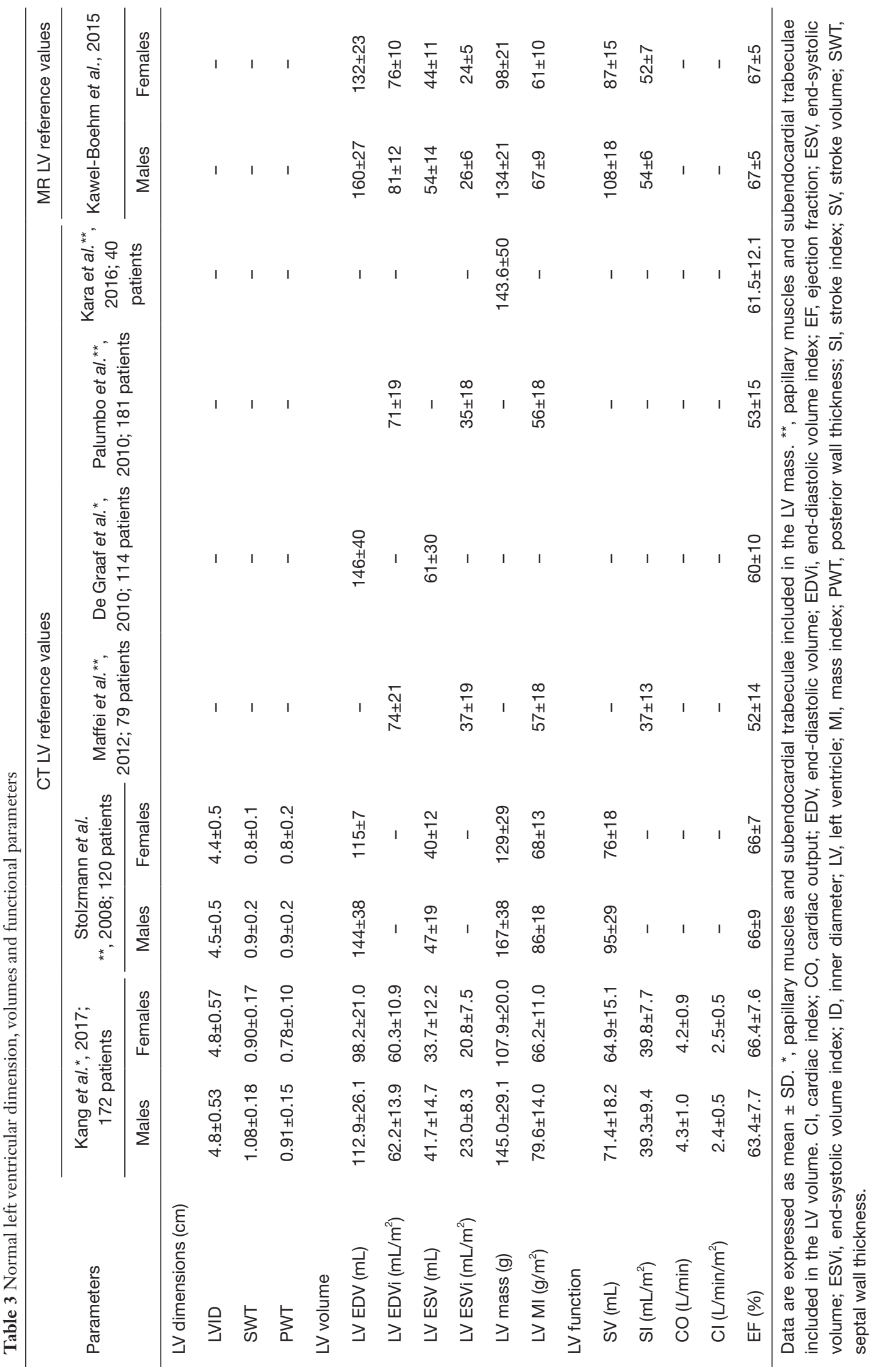



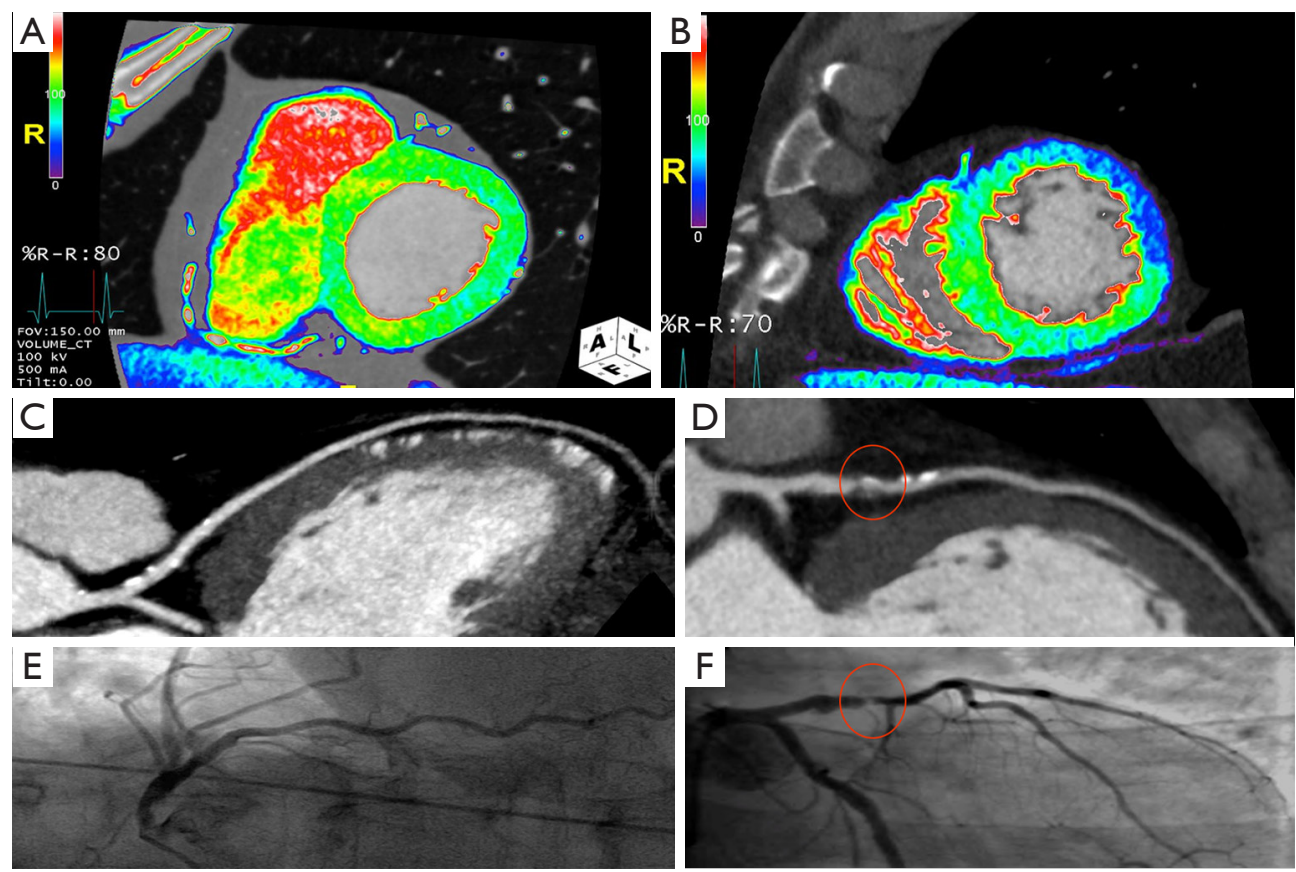

Figure 4 First-pass myocardial perfusion imaging evaluation during angiographic coronary evaluation (curved-MPR LAD image) and ICA control. Normal LV first pass perfusion related to calcific non obstructive CAD of LAD (A, CCTA short axis color map; C, LAD curvedMPR; E, ICA) and perfusion defect of the anterior and anterolateral LV wall related to LAD obstructive mixed coronary plaque with ICA control (B, CCTA short axis color map; D, LAD curved-MPR; F, ICA). LAD, left anterior descending coronary artery; CAD, coronary artery disease; ICA, invasive coronary angiography; red circle, proximal LAD severe obstructive coronary plaque.

defects can be directly visualized as hypoattenuated or nonenhancing regions (14).

Delayed-enhancement CT facilitates the evaluation of $L V$ myocardial viability or scarring. A delayed scan is usually performed after a variable period of 5-15 minutes following the initial administration of contrast medium decreasing the tube voltage (100 or $80 \mathrm{kVp}$ ) and using prospective ECG triggering to reduce the radiation exposure. Delayed hyperenhancement is attributed to the slow washout of iodinated contrast media from the region of infarcted myocardium (15). Gerber et al. showed that delayed imaging can be used to quantify infarct size which is strongly correlated with CMR-based imaging (16).

As with ultrasounds, CCTA can offer morphological screening, particularly in patients with concomitant cardiomyopathy and CAD. End-diastolic chamber dimensions and wall thickness can be precisely measured and dilated (DCM) or hypertrophic cardiomyopathies (HCMs) identified.

Even if CCTA is not included in screening methods in the evaluation of cardiomyopathies and CAD in relation to the radiation exposure, it represents a possible modality useful in selected patients.

CCTA can discriminate between ischaemic and nonischaemic causes in dilated phenotype. DCM is characterized by ventricular dilatation and hypertrabeculation, global LV wall thinning, impaired systolic function and septal mid-wall delayed-enhancement pattern in the absence of CAD.

CCTA plays a potential role in the assessment of HCM (Figure 5) based on key morphological features (asymmetric hypertrophy involving the septum and myocardial crypts characterized by a narrow, deep invagination within the myocardium localized predominantly in the inferoseptal LV myocardium), the presence of "patchy and nodular" delayed-enhancement (the hallmark of HCM) as well as $\mathrm{CAD}$ evaluation simultaneously. In addition, retrospective ECG-gating allows to assess systolic anterior motion (SAM) of the MV in hypertrophic obstructive cardiomyopathy (HOCM).

While the Task Force Criteria do not consider CT (17), LV myocardial fat in arrhythmogenic right ventricular cardiomyopathy (ARVC) appears as a wedge-shaped or bandlike configuration in the subepicardial LV-free wall on CT. 

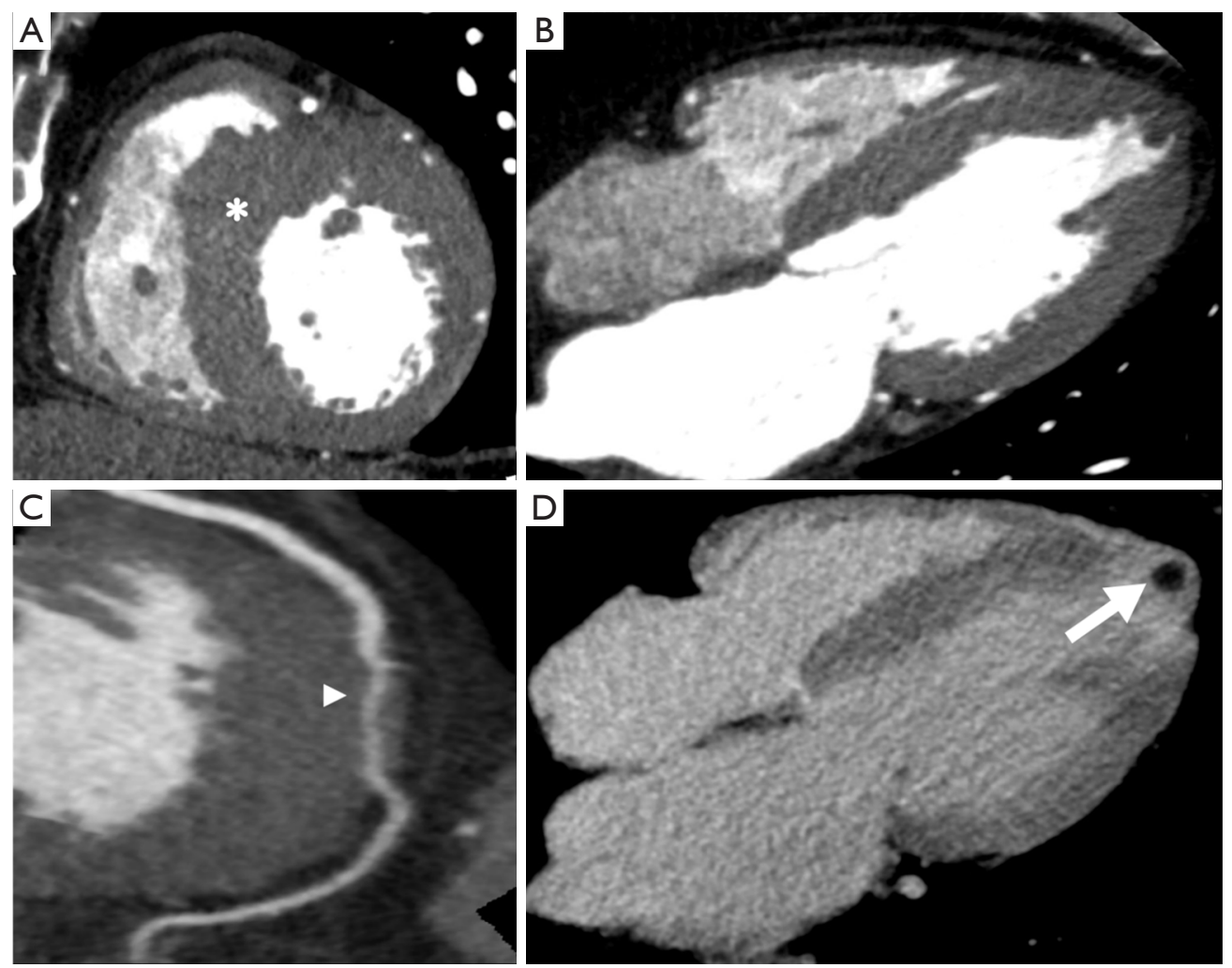

Figure 5 Diastolic short-axis (A) and four-chamber (B,D) cardiac-gated CT images show asymmetric septal HCM (asterisk) in a 51-year-old man with apical thrombus (arrow) and left distal anterior descending coronary myocardial bridge (C, arrowhead). HCM, hypertrophic cardiomyopathy.

In addition, CT is able to characterize fat infiltration of the RV (Figure 6) and left subendocardial wall fat related to myocarditis scar (18).

In patients with acute chest pain, CCTA may be used to evaluate coronary artery (CA) calcium score and exclude CAD. In the ischaemic cardiomyopathy, noninvasive fractional flow reserve-computed tomography angiography $\left(\mathrm{FFR}_{\mathrm{CTA}}\right)(19)$ is evaluable in addition to regional $\mathrm{LV}$ wall thinning (end-diastolic wall thickness $<5.5 \mathrm{~mm}$ ) and wall motion abnormality, subendocardial/transmural myocardial perfusion defects or scar, fatty replacement or myocardial calcification in the culprit CA territories possibly associated with apical aneurysm and intraventricular thrombus formation (Figure 5).

On CCTA images a LV aneurysm is a thinned scarred outpouching of the myocardium with a broad neck characterized by paradoxical expansion or dyskinesia while a LV pseudoaneurysm (or false aneurysm) is an akinetic outpouching caused by free wall rupture limited by an overlying adherent pericardium without a myocardial layer and characterized by a neck narrower than the diameter of the aneurysm, an abrupt transition from normal myocardium to aneurysm and a distinct discontinuity of the ventricular wall (20).

In acute myocarditis, subepicardial delayed-enhancement CT correlates with CMR based on areas and degrees of involvement. A reduced tube voltage increases the contrast of iodine and simultaneously lowers radiation exposure (21). CCTA also reveals the typical delayed enhancement (multifocal, intramural with spotty distribution) in cardiac sarcoidosis, as well as mediastinal lymphadenopathy and involvement of other organs (22).

\section{Right ventricle}

The evaluation of the volumes and function of the RV is crucial in many forms of congenital and acquired heart disease. In complex CHD, such as tetralogy of Fallot, the assessment of the RV volumes and function is important for clinical and surgical decision making. RV function is also a 

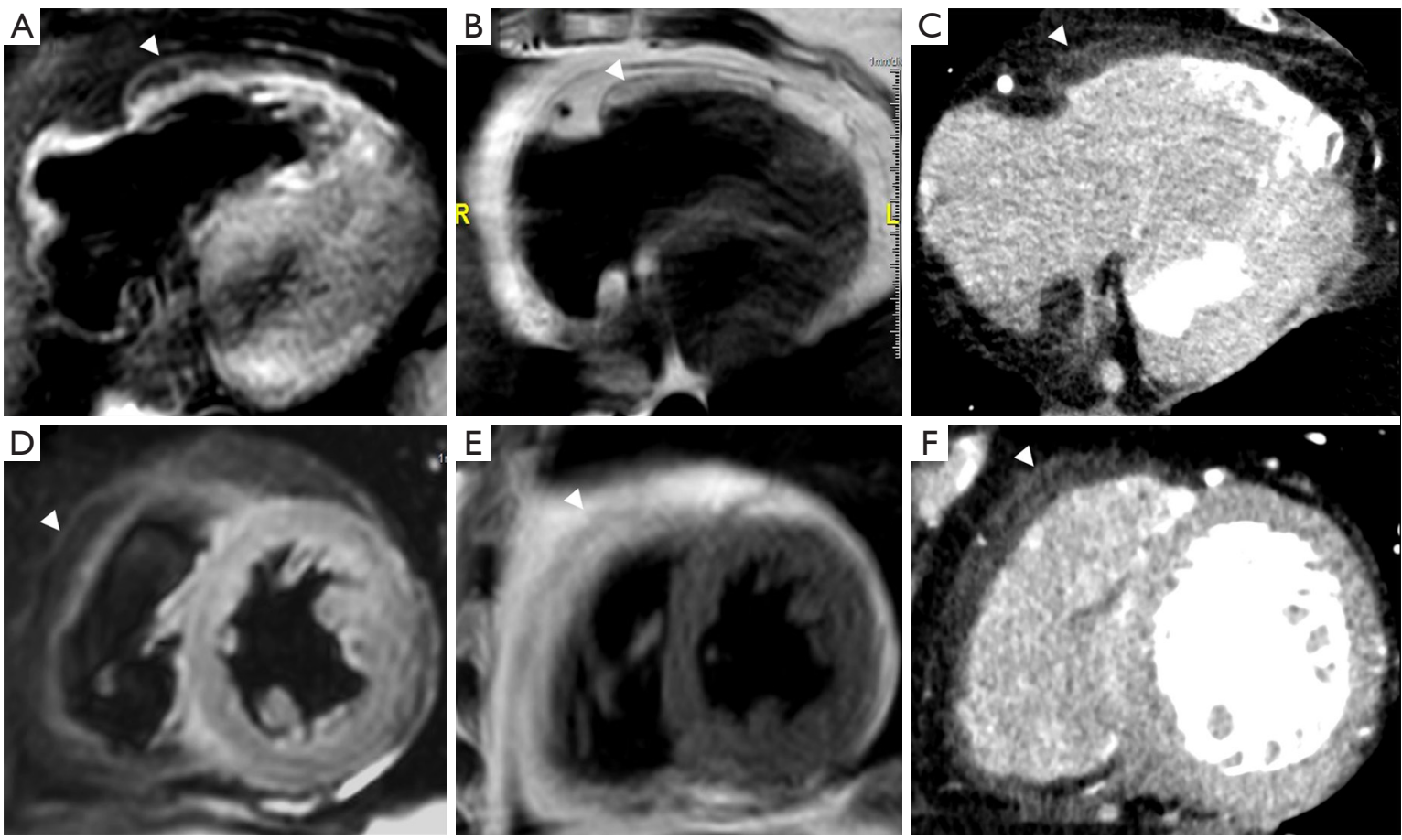

Figure 6 Long-axis and two-chambers short axis view CMR T2-STIR (A,D), dark-blood T1-weighted (B,E) and CCTA (C,F) showing $\mathrm{RV}$ adipose infiltration (arrowhead). CCTA, cardiac computed tomography angiography; RV, right ventricle; CMR, magnetic resonance imaging.

prognostic marker in pulmonary hypertension, pulmonary embolism and other myocardial diseases. CMR is considered the gold standard for the evaluation of the RV. However, in patients with contraindication to CMR, such as PM or ICD implant, CCTA represents a valid cross-sectional alternative. As a matter of fact, assessment of RV function by CCTA has been validated in multiple studies and provides comparable information with good accuracy and reproducibility using CMR as the reference standard (23) (Table 4). Moreover, CCTA can assess most of the major or minor criteria of the original task force criteria for the diagnosis of ARVC $(17,24,25)$.

\section{Valvular apparatus}

The fibrous rings of the heart are mainly located on the heart valve plane and anchor the muscle bundles constituting the wall of the atria and ventricles. They contain the mitral, tricuspidal, aortic and pulmonary valves. The space between the mitral and tricuspid valves and aorta forms the right fibrous trigone which is crossed by the atrioventricular bundle of the conduction system of the heart (Figure 7).

\section{Aortic valve}

The aortic valve (AV) is a trifoliate structure supported by a fibrous skeleton, linked with the anterior leaflet of the MV. The three valve cusps are thin and symmetric leaflets of fibrous tissue that open into their respective sinuses of Valsalva during the systolic phase. The commissures are the joining points between the valve cusp attachments and the root. The aortic root is a complex structure, schematically outlined with three parallel virtual rings (sinotubular junction, ventricular-aortic junction and basal attachment of AV leaflets) and one crown-shaped line (representing the attachment of each cusp to the respective Valsalva sinus, sometimes called "surgical annulus") (26). The virtual basal ring is the virtual ring defined by the three anchors at the nadir of each aortic cusp attachments, usually inhomogeneous because of calcifications.

CCTA represents the reference standard for the anatomic assessment of the aortic valve and aortic root in relation to its intrinsic high accuracy related to characteristics in planning AV intervention such as high spatial resolution.

The numeric variants of the leaflets and the fusion of the commissures (Figure 8) are the most common anatomical abnormalities of the AV and clearly shown by 


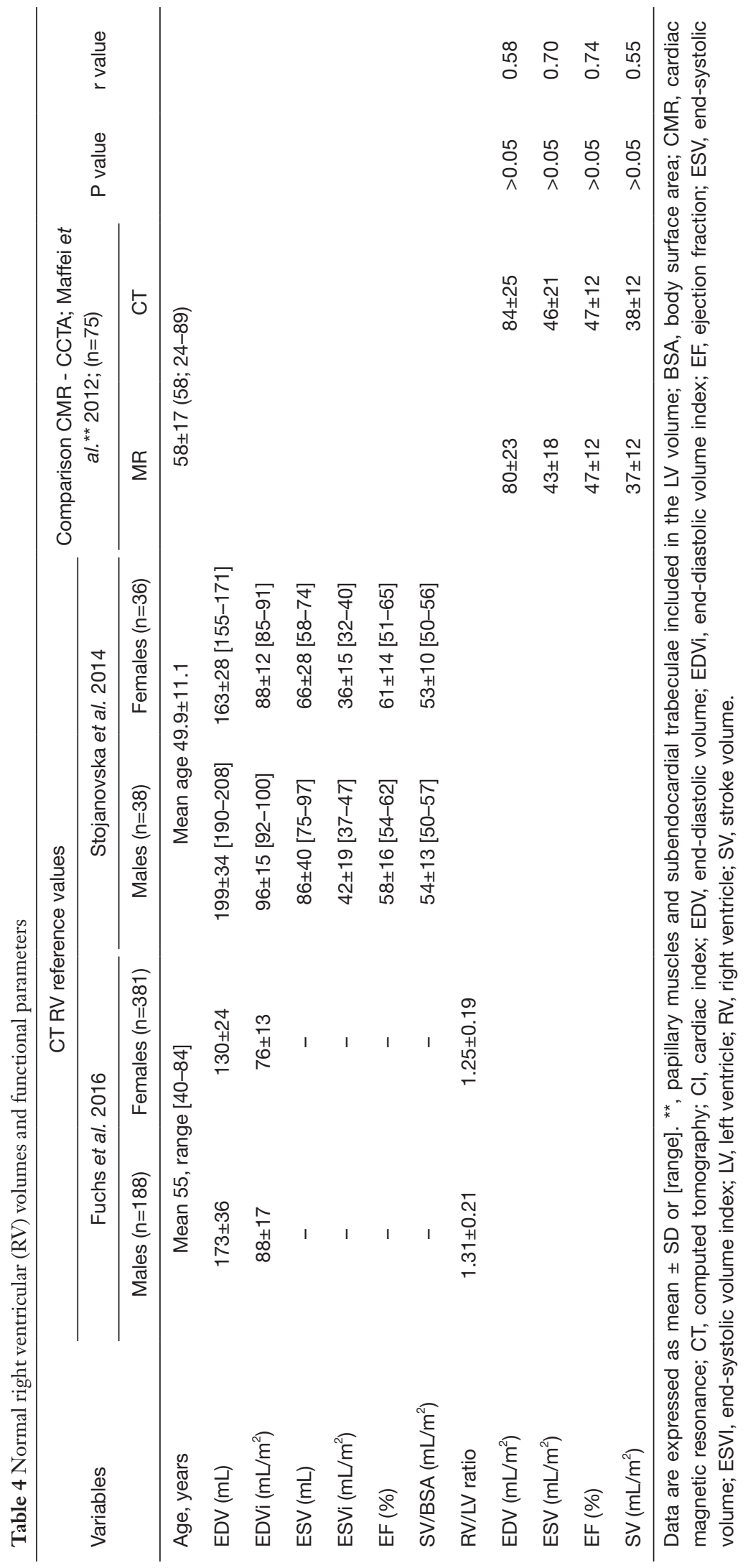




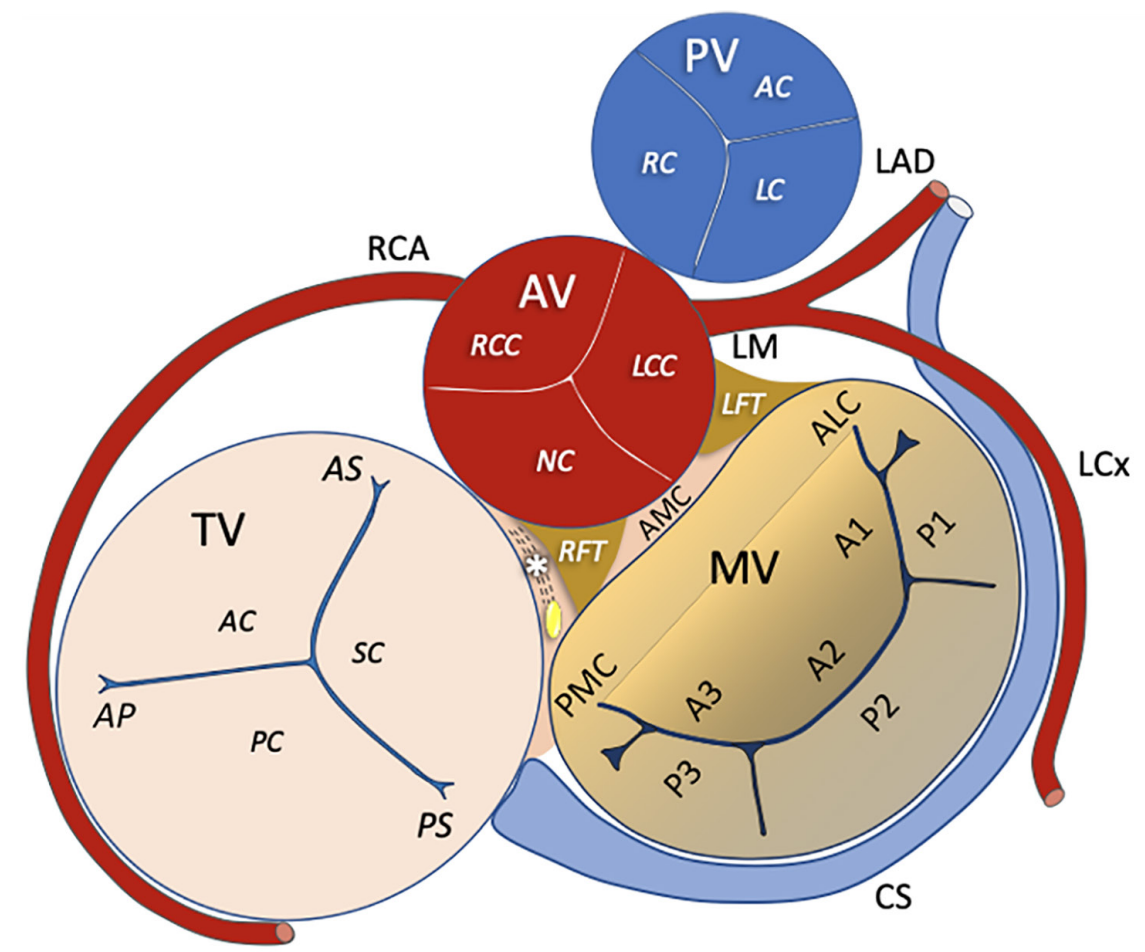

Figure 7 Base of the heart showing the four cardiac valves. Mitral valve (MV) anatomy showing the D-shaped mitral annulus at the atrioventricular junction, which has a 3D saddle-shaped, nonplanar geometric structure with a greater inter-commissural than mediolateral diameter. MV has two distinct asymmetric leaflets: the posterior (or mural) leaflet, which is narrow and extends two-thirds around the left atrio-ventricular junction; the anterior (or aortic) leaflet, which is much broader and thicker and comprises one third of the annular circumference. A distinct feature of the anterior leaflet is the fibrous continuity with the left and noncoronary cusps of the aortic valve and with the interleaflet triangle between aortic cusps abutting the membranous septum (aortic-mitral curtain). Despite the posterior leaflet appears smaller compared to the anterior leaflet, it has a larger surface (approximately $5 v s .3 \mathrm{~cm}^{2}$, respectively). For descriptive purposes, according to Carpentier nomenclature of the MV leaflets structure: $\mathrm{A}=$ anterior, $\mathrm{P}=$ posterior. Two clefts of the $\mathrm{P}$ leaflet divide it into three scallops (segments) along the free edge: P1 = lateral, P2 = middle, P3 = medial; the opposing virtual segments of the A leaflet are labelled A1, A2 and A3. The relationship is also shown to the aortic valve (AV), pulmonary valve (PV) and tricuspid valve (TV). The tricuspid valve has an elliptical and nonplanar shape, with the posteroseptal (PS) portion "lowest" (toward the apex), with three roughly triangular cusps, which when considered relative to their positions in the body, are properly described as being septal (SC), anterior (AC), and posterior (PC). ALC, anterolateral commissure; PMC, posteromedial commissure; AMC, aorto-mitral curtain; RFT, right fibrous trigone; LFT, left fibrous trigone; yellow dot, atrioventricular bundle; NC, noncoronary cusp; LCC, left coronary cusp; RCC, right coronary cusp; AS, antero-septal commissure; PS, posteroseptal commissure; AP, antero-posterior commissure; AC, anterior cusp; SC, septal cusp; PC, posterior cusp; RC, right cusp; LC, left cusp; RCA, right coronary artery; LM, left main coronary artery; LAD, left anterior descending coronary artery; LCx, left circumflex coronary artery; CS, coronary sinus; *, atrio-ventricular part of membranous septum.

CCTA examinations. The unicuspid variant, frequently associated with early severe aortic stenosis occurring in infancy or childhood and with ascending aortic dilatation is rare (27). Bicuspid aortic valve (BAV) is the most common congenital heart disease (CHD). It is often associated with other congenital cardiovascular abnormalities or genetic disorders (28). Several classifications of BAV exist, based on the presence/absence of raphes, on cusp fusion, size and position, and on the number of sinuses (29). Quadricuspid aortic valves are extremely rare variants, generally associated with incomplete coaptation of the cusps $(30,31)$.

Even if the grading of aortic stenosis severity should be performed by CMR (32), the visualization of the aortic valve and aortic root during the cardiac cycle is also shown 

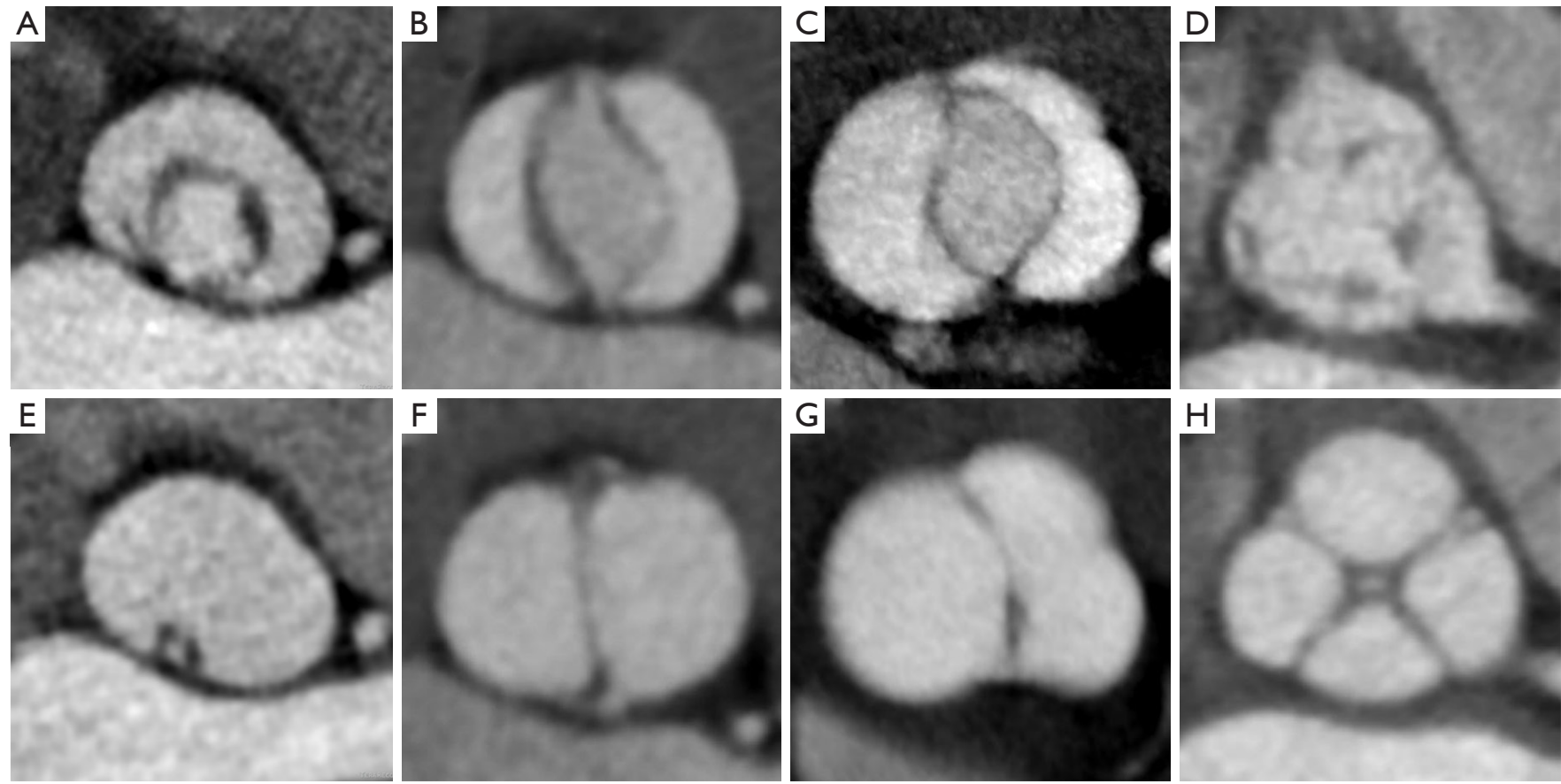

Figure 8 Aortic valve morphologies. Unicommissural unicuspid valve with an eccentric loophole-shaped orifice (A, systole; E, diastole); bicuspid valve without raphe having two cusps and commissures (B, systole; F, diastole); bicuspid valve with raphe containing two cusps and commissures (C, systole; G, diastole); quadricuspid valve with incomplete leaflet coaptation due to insufficiency (D, systole; H, diastole).

by CCTA. In this condition, it is possible to search for the correct evaluation plan and measure the maximum residual systolic area at the level of the aortic limbs.

\section{Mitral valve}

The MV is a complex anatomical and functional apparatus which can be evaluated by CCTA showing the mitral annulus at the atrioventricular junction, which has a $3 \mathrm{D}$ saddle-shaped, nonplanar geometric structure with a greater inter-commissural than mediolateral diameter $(33,34)$, and the posterior (or mural) leaflet, which is narrow and extends two-thirds around the left atrioventricular junction and the anterior (or aortic) leaflet, which is much broader and thicker and comprises one third of the annular circumference (Figure 9).

The chordae tendineae can be shown according to their attachment $(33,34)$.

There are usually two dominant groups of papillary muscles (PMs), named anterolateral and posteromedial PMs, which are located in the mid to apical segments of the left ventricular wall and are aligned with the MV commissures (33). A considerable variation in the number, shape, and patterns of PMs (i.e., single PM with or without multiple heads or multiple PMs) is reported (32). Most frequently they have a conical, cylindrical or a broadapexed shape, and arise from a broad, mesh-like, network of trabeculation (i.e., named "cypress root") (35-37). The PMs contraction during systole tightens chordae tendineae and prevents leaflet prolapse.

Typically, the CCTA acquisition for valvular workup involves full cycle acquisition. CCTA due to its high isotropic spatial resolution can accurately assess the $\mathrm{MV}$ morphology [leaflet thickening, calcification, or mitral annular calcification (MAC)], and the abnormalities of the MV apparatus (i.e., degenerative prolapse, flail, or infective endocarditis) (38).

CCTA may allow reliable evaluation of MV stenosis and its severity grade. A good correlation, although with systematic overestimation, of CT-derived MV planimetric area with that of Doppler transthoracic echocardiography (TTE) and cardiac catheterization has been demonstrated (39). CCTA plays an important role in defining the landing zone for transcatheter MV intervention, offering highly reproducible evaluation of the $3 \mathrm{D}$ geometry of the MV annulus (40). CCTA provides a detailed and clear definition of the extent and severity of annular calcium, 

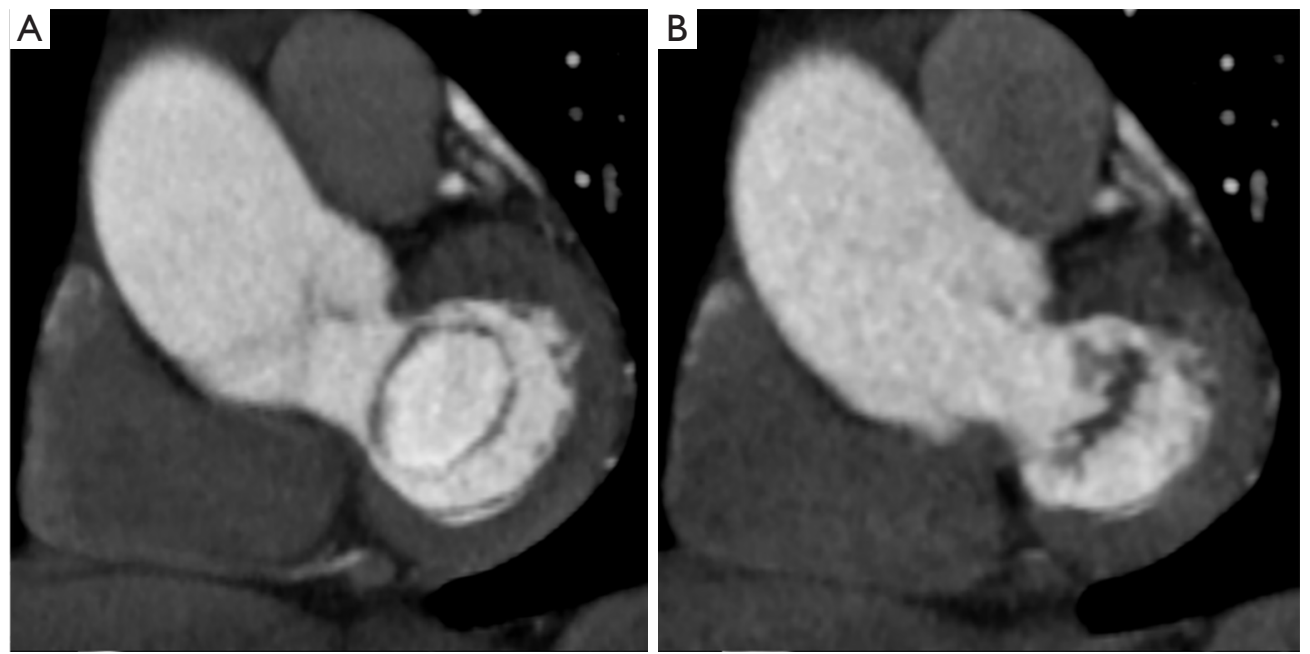

Figure 9 Short-axis view of the normal mitral valve during diastole (A) and systole (B).

which can help determine device suitability (40). Finally, CCTA offers the substantial advantage of the accurate preoperative assessment of $\mathrm{CAD}$ in patients with valvular heart disease when surgery or an intervention is planned. Owing to its high negative predictive value, current guidelines recommend CCTA with a Class IIa, LoE C, in patients with low probability of CAD (41).

\section{Tricuspid valve}

Clear depiction of the right heart requires homogeneous enhancement of the structures around the tricuspid valve (TV) annulus. Most ECG-gated CCTA techniques with a modified contrast injection in order to permit the right heart examination (i.e., high flow contrast bolus followed by a $50 \%$ diluted contrast and saline chaser bolus) can provide good-quality motion-free images of the right chambers without flow artifacts that can usually be present in the right atrium and RV inlet (Figure 10).

Nowadays, CCTA has gained an important role in preprocedural planning for percutaneous TV interventions, similar to aortic and mitral procedures (42). CT is important for several preprocedural steps of percutaneous TV repairing procedures, providing complementary information to echocardiography and fluoroscopy, especially for interventions targeting the tricuspid valve annulus (43), analyzing the annular structure and dilatation, the quality and the amount of annular tissue and its relationship with the right coronary artery (RCA) in different regions of the TV. Furthermore, sizing the inferior vena cava (IVC) is of utmost important for the planning of some percutaneous procedures. The normal morphology of the TV can be clearly depicted by CCTA even if it has an elliptical and nonplanar shape $(44,45)$. During the cardiac cycle, the annulus area changes by approximately one-third, with the diameter changing by one-fifth $(46,47)$. ECG-gated CT reveals this unique tricuspid morphology of the annulus, along with its temporal changes in different phases of the cardiac cycle.

\section{Pulmonary arteries}

The role of CCTA scan in the evaluation of the pulmonary artery is well established (48). Thanks to the excellent isotropic spatial resolution and a multiplanar reconstruction, CCTA allows accurate measurements of pulmonary arteries (PAs) and pulmonary branches in CHD and also in acquired Heart disease. Accurate evaluation of PAs is a frequent target in the imaging of CHD before and after repair. In this population, when echocardiography is non exhaustive and CMR is contraindicated or considered a high risk for the time of the scan and sedation, CCTA is increasingly used. Moreover, several studies reported the role of CCTA for interventional planning of complex pulmonary anatomy (49) and for complex lung lesions such as intralobar or extralobar pulmonary sequestration (50). CCTA is widely used for evaluation of lung diseases, in this setting published studies reported that an enlarged PA and the PA/aortic diameter ratio may predict pulmonary hypertension, with CT-based cutoff values ranging respectively from 28.6-33.2 $\mathrm{mm}$ and from 0.84 to 1.4 (51). CT Pulmonary Angiography is also an already established technique in patients with 

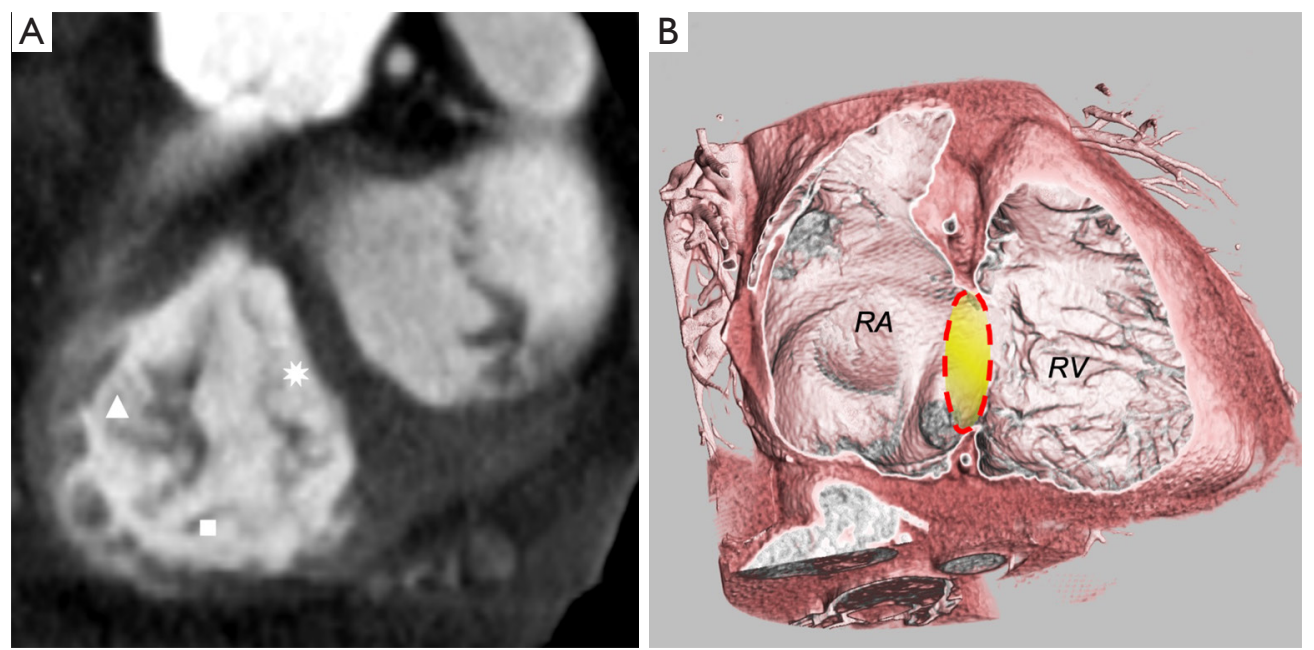

Figure 10 Short-axis view (A) at the level of the tricuspid annulus (TA) in patient with functional regurgitation localization of the anterior $(\boldsymbol{\Delta})$, septal (*) and posterior (-) leaflets and 3D-VR cut plane showing the TA plane (B).

suspected pulmonary embolism and is a crucial component in commonly used clinical diagnostic algorithms (52).

\section{Pericardium}

The pericardium is a double walled fibroserous sac (a small cavity filled with up to $60 \mathrm{~mL}$ of serous fluid) composed of the visceral pericardium and parietal pericardium. Two pericardial lines of reflection localized around the aortopulmonary vascular pedicle and the venous pole of the heart determine the presence of different pericardial sinuses and recesses (53). On CCTA images the pericardium appears as a thin soft tissue line enveloping the heart. It extends superiorly from the great vessels and inferiorly to the diaphragm and is best visualized along the $\mathrm{RV}$ (54). Its thickness ranges from 1 to $2 \mathrm{~mm}$, although it is considered normal up to $4 \mathrm{~mm}$. Several diseases may affect the pericardium. The most common CCTA finding relates to the presence of pericardial effusion; depending on attenuation values [Hounsfield unit (HU)], it can be distinguished in exudative or hemorrhagic (55). Malignant pericardial effusion should be suspected in case of hemorrhagic effusion with pericardial nodular lesions. In the setting of a rapidly developing effusion, a cardiac tamponade may occur, in which pericardial collection causes the compression of the right cardiac chambers and the bowing of the interventricular septum (IVS), resulting in diastolic impairment (56). Acute inflammation of the pericardium (pericarditis) results in pericardial thickening $(>4 \mathrm{~mm})$ and enhancement which may be preferably evaluated during the portal venous phase rather than during the arterial phase (57). One more typical finding is pericardial calcifications, which constitutes an end-stage response to some injuries (58). They appear as linear or patchy hyperdensities that may encase the entire heart, resulting in constrictive pericarditis and subsequent impaired ventricular filling and right heart failure. CCTA can depict the extent of calcium amount and assess complications such as the intramyocardial calcium invasion, which may hinder the success of a pericardiectomy (48). Other less common pericardial diseases include pericardial cysts and diverticula, the epipericardial fat pad necrosis and benign and malignant tumors.

\section{Right and left atrium}

CCTA is an option for atria reconstruction and imageintegration for real-time navigation in electrophysiological procedures and after inadvertent acute or late complications. CCTA can be useful in pre-procedural planning of CRT implantations showing coronary sinus (CS) anatomy, barriers at the ostium, challenging access in the vessel and collaterals and anatomical variants. CCTA may offer a non-invasive optimal panoramic view of the right atrium (right appendage, crista terminalis, septum, cavotricuspid isthmus anatomy, prominent thebesian valve) alongside $3 \mathrm{D}$ transesophageal echocardiography (TOE) and intracardiac echocardiography (ICE) (59-61) (Table 5).

CCTA has a dominant role in left atrial (LA) procedures. Number of pulmonary veins, pattern of branching, ostial 
Table 5 Atrial chambers characteristics

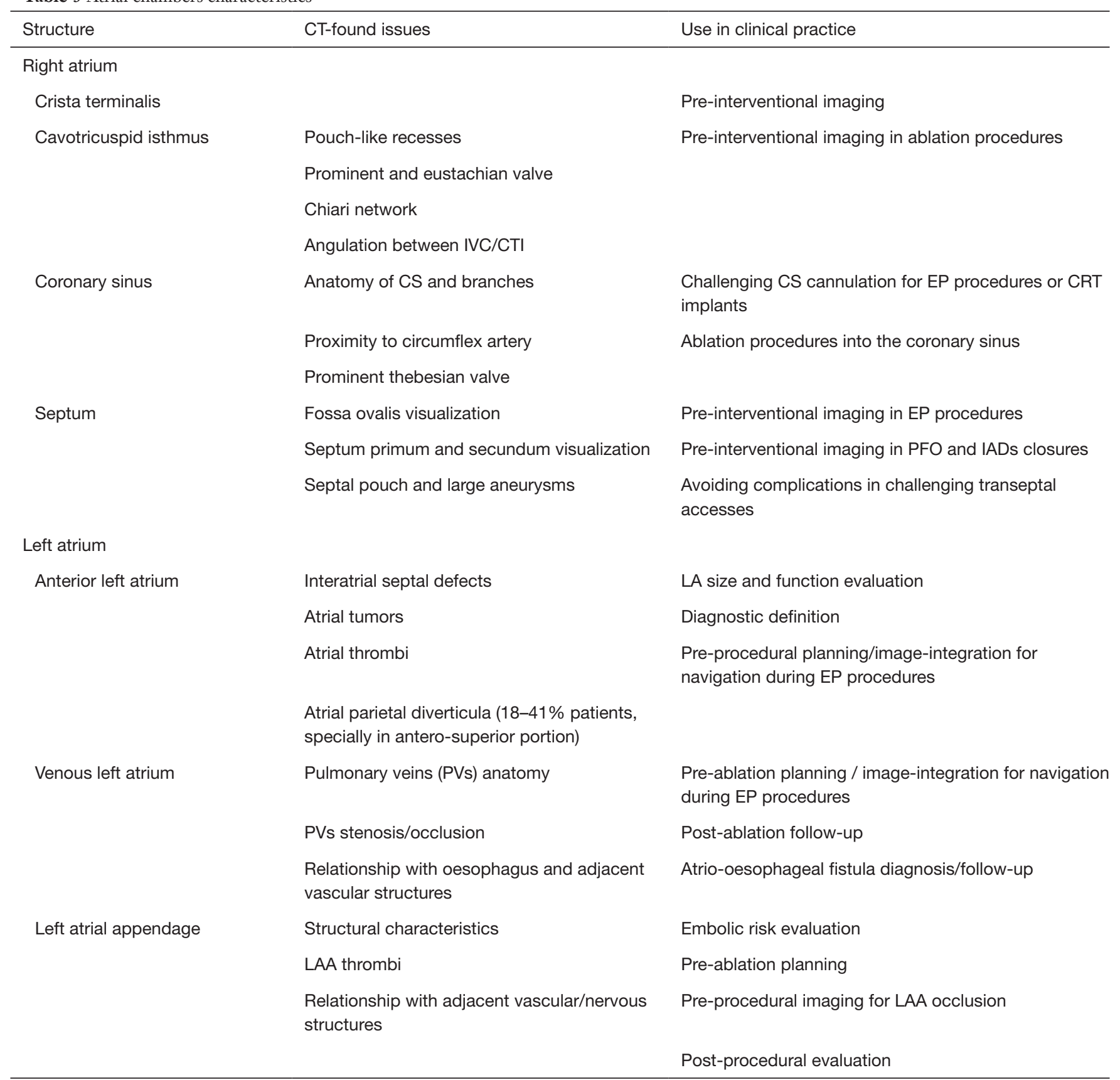

CS, coronary sinus; EP, electrophysiology; CRT, cardiac resynchronization therapy; IVC, inferior vena cava; CTI, cavotricuspid isthmus; PFO, patent foramen ovale; IAD, interatrial septal defect; LAA, left atrial appendage; LA, Left atrium.

morphology and measurements, and more importantly anomalous pulmonary vein variants, accessory pulmonary veins or anomalous pulmonary venous drainage can be easily identified by CCTA compared with other modalities such as TOE and ICE. Pulmonary vein stenosis is nowadays an infrequent, yet known complication following catheter ablation procedures. In patients with symptoms suggestive of pulmonary venous obstruction, serial CT assessment of pulmonary veins allows for detection or exclusion of stenosis (61-63). Pre-procedural imaging showing presence 
of parietal diverticula (typically in anterosuperior LA portion) can make ablation procedures safer. While TOE is considered the gold standard for the detection of left atrial appendage (LAA) thrombi prior to ablation procedures, several studies have shown a high diagnostic accuracy of CCTA to detect LAA thrombi (61). High definition imaging of LAA and adjacent vascular and nervous structures is relevant in LAA plugging procedures allowing correct device sizing $(61,62)$. Furthermore, anatomical relationship between venous LA and posterior wall to the oesophagus can be easily identified and is beneficial in the risk reduction of lethal complications such as atrioesophageal fistula caused by high-energy application during ablation procedures (61-62).

\section{Coronary artery anatomy and CAD}

Reliable interpretation of coronary angiography by CCTA requires a sophisticated understanding of: normal coronary artery (CA) and cardiac anatomy; the pathophysiology of coronary atherosclerosis and other abnormalities, including congenital anomalies; the characteristic appearance of CA and cardiac lesions on CCTA with and without contrast medium (63); the technology and limitations of CCTA; the use of 3-dimensional cardiac-specific interpretation software; and the ability to identify and overcome image artifacts in the available image data set $(64,65)$. The currently recommended training process to attain competency in interpretation has been outlined in previous medical specialty society statements $(66,67)$.

The coronary segmentation classification recommended by the American Heart Association (AHA) is used to create a CCTA reporting system (67), thus facilitating the communication between physicians. The AHA classification divides the coronary arteries into 15 or 16 segments (Figure 11). The coronary tree should be initially examined for the course and branching of the main coronary vessels and sub-branches. The Society of Cardiovascular Computed Tomography (SCCT) guidelines recommends an axially based model of coronary segmentation, adapted for coronary CTA (65). This model varies from the standard AHA segmentation in the following ways: a left posterolateral branch is identified as segment 18 , and a ramus intermedius branch has been added as segment 17 .

Congenital CA anomalies are of major significance due to their association with myocardial ischaemia and sudden death and their prevalence ranges from $0.21 \%$ to $5.79 \%$ (68) (Figure 12). The most commonly used classification of CA anomalies is based purely on anatomical considerations $(69,70)$. Anatomy and Pathology Working Group of the European Society of Cardiology published a position statement in order to provide a classification linked to coronary embryonic developmental mechanisms and CA congenital anomalies (70) (Figure 13).

\section{Perioperative assessment of congenital heart defects}

In recent years, CCTA has acquired an increasingly important role in the diagnosis and preoperative planning of congenital heart defects $(71,72)$ and in adult surgical reoperation in defining cardio-vascular-sternal relationships. In the scenario of multiple surgical procedures, the development of tenacious cardio-sternal adhesions represents an element of considerable bleeding risk during the chest reopening phase. Therefore, CCTA imaging becomes a fundamental aid in guiding the reopening procedure allowing to adopt the strategy with less surgical risk (73).

For the correct preoperative diagnostic definition of partial and total anomalous pulmonary venous connections, echocardiography is often insufficient to guide surgical planning and CCTA becomes essential. Especially in the total and mixed forms of anomalous pulmonary venous connection, the questions that CCTA must answer are the anatomy of the veins confluence and the course and draining site of the venous collector (74). The choice of the best surgical technique is the consequence of a perfect and exhaustive preoperative anatomical definition. The correct knowledge of the normal and CA anomalies should be examined with regard to their origin, course, and relationship to the cardiac chambers, aorta, pulmonary artery, and interventricular septum together with the mediastinal and thoracic structures in the operative planning. Consequently, CCTA allows to verify the surgical result by highlighting all anatomical details of the surgical techniques employed (75).

Even the group of conotruncal anomalies such as complex transpositions of great vessels (TGA) becomes an area of increasing application of the most advanced CCTA methods (76). Many complex surgical procedures actually adopted for anatomical correction of complex TGA can be indicated and guided by advanced modalities of CCTA imaging $(76,77)$.

Vascular rings are a group of aortic arch defects causing tracheoesophageal compression at different levels depending on the anatomical type of defect. The key role of CCTA and its dynamic modalities in diagnosis, anatomical 
Right coronary artery (RCA)
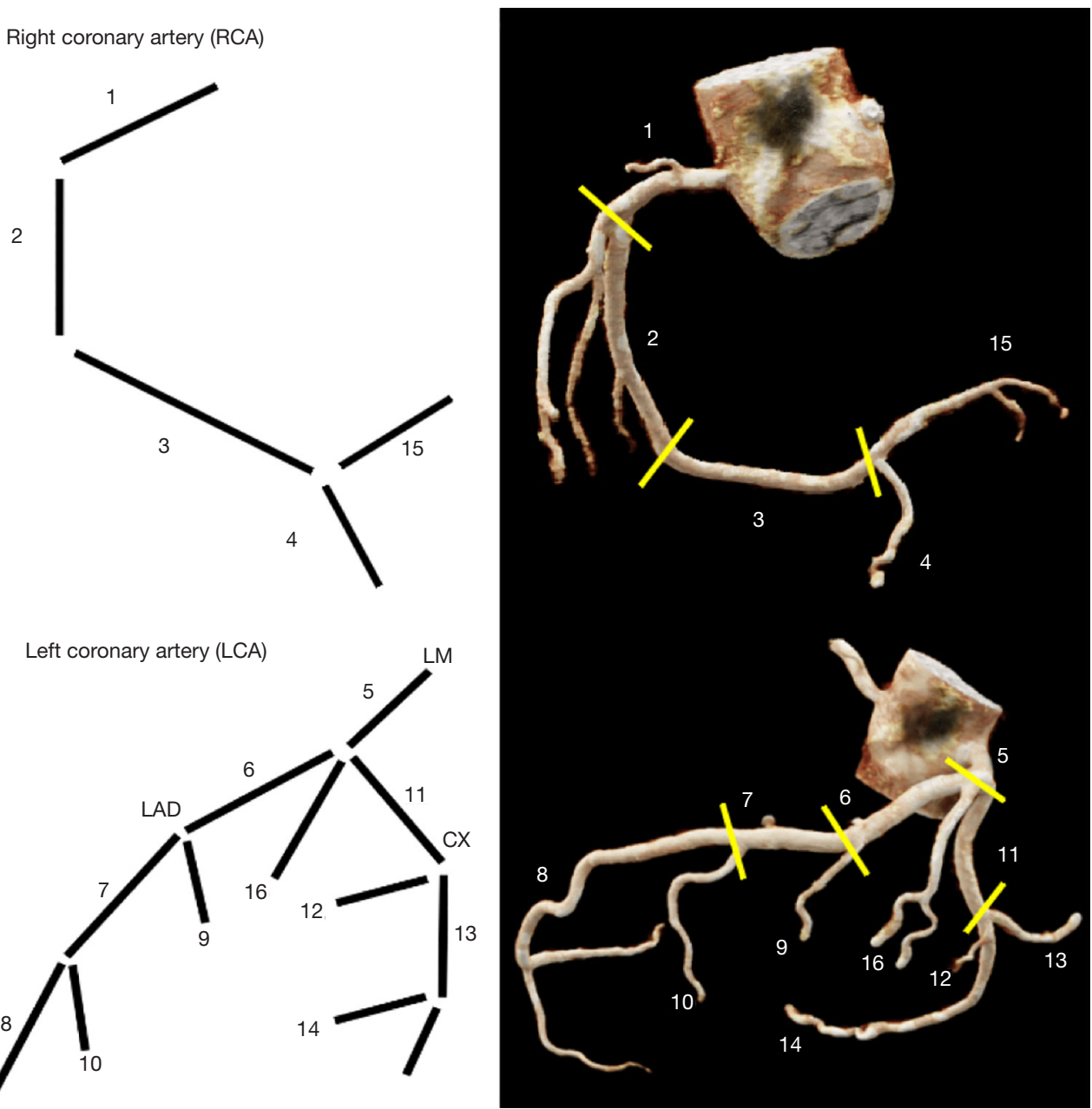

Figure 11 According to AHA classification, Volume Rendering Image depicts the anatomy and the same classification of the right coronary artery (RCA) and of the left coronary artery. The RCA, which arises from the right coronary sinus (RCS), is conventionally divided into three segments: the proximal (segment 1), the mid (segment 2), and the distal (segment 3), which runs in the posterior right atrioventricular groove from the acute margin of the heart to the origin of the posterior descending branch (PD, segment 4). The posterolateral branch (PL, segment 15) usually arises from the RCA in the typically right-dominant circulation as a terminal branch along with the PD but can less commonly arise from the left circumflex (LCX) and rarely from the left anterior descending artery (LAD). The left main coronary artery (LM, segment 5) extends from the ostium of the left coronary artery (LCA) to the bifurcation in LAD and LCX branches. The LAD, which runs in the anterior interventricular groove, is conventionally divided into: the proximal tract (segment 6), including the origin of the first diagonal branch (segment 9); the mid tract (segment 7), which extends to include the point where the LAD forms an angle and generally it coincides with the second diagonal branch (segment 10); the distal or apical tract (segment 8), which extends beyond the apex. The LCX is divided into proximal (segment 11, including the origin of the first obtuse marginal branch) and distal (segment 13, running in the left atrioventricular groove) segments. The branches for the obtuse margin of the heart which supply the lateral wall of the left ventricle are called segment 12 and segment 14. Segment 16 indicates a possible additional branch known as the "intermediate" (IM), which originates from the LM and runs along the anterolateral wall of the left ventricle. AHA, American Heart Association. Yellow line, coronary segment demarcation. 

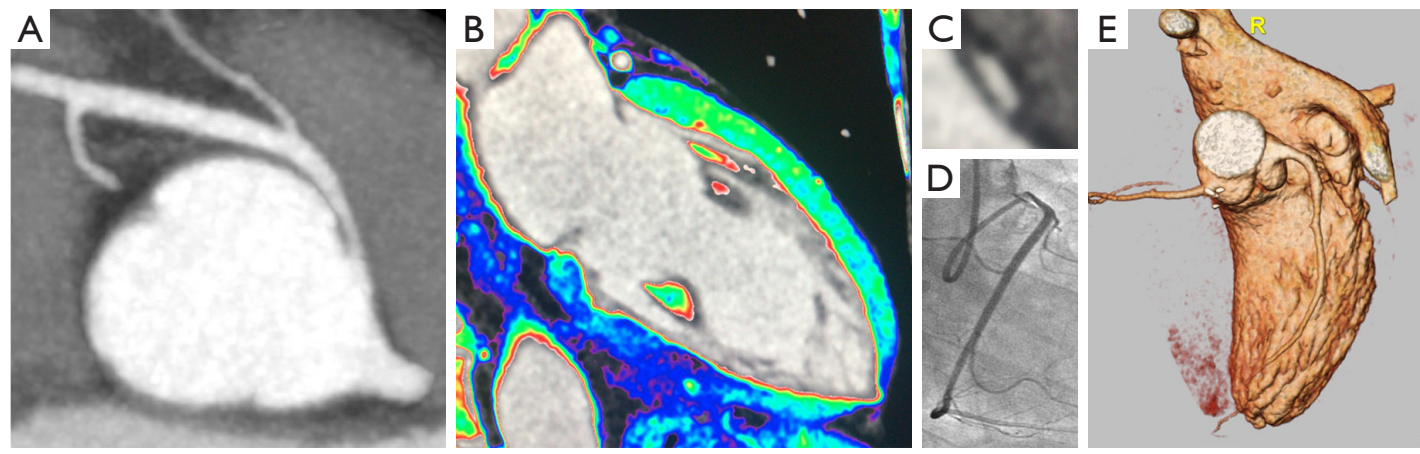

Figure 12 Anomalous origin of the RCA from the left sinus of Valsalva and interarterial course (A), causing ischemia during exercise and hypoperfusion of the inferior septal wall of the LV during rest angiographic CT acquisition (B), color-coded in blue-violet and "slit-like ostium" at CT and ICA (C,D). After surgical repair (E) no more chest pain and related ischemia were observed. RCA, right coronary artery; $\mathrm{LV}$, left ventricle; ICA, invasive coronary angiography.

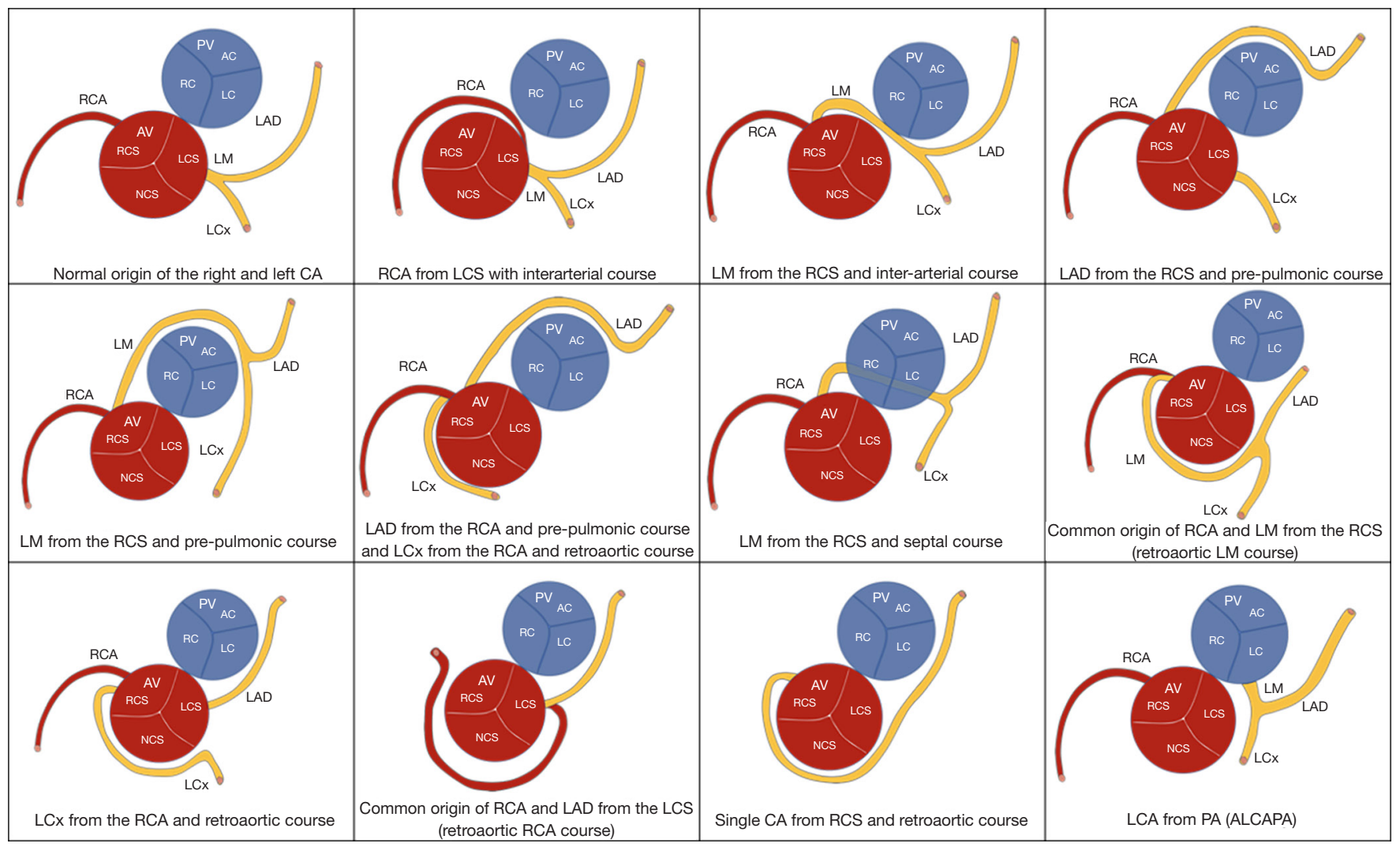

Figure 13 Normal anatomy and major congenital anomalies of CA-truncal connection. Anomalous CA connection to the aorta: RCA from the left coronary sinus (LCS) with interarterial course; LCA from the RCS with interarterial course; LAD from the RCS with prepulmonic course; LCA from the RCS and prepulmonic course; LAD from the RCS with prepulmonic course and LCX from the RCA and retroaortic course; LCA from the RCA with interseptal course; LCA from the RCS or single CA with LCA with retroaortic course; LCX from the RCS or RCA with retroaortic course; single CA from the LCS with retroaortic course of RCA; single CA connected to the RCS with retroaortic course; LCA from the pulmonary artery. RCA, right coronary artery; LCA, left coronary artery; LM, left main coronary artery; LAD, left anterior descending coronary artery; CA, coronary artery; LCx, left circumflex coronary artery; LM, left main coronary artery; RCS, right coronary sinus; LCS, left coronary sinus; NCS, non-coronary sinus; PA, pulmonary artery; PV, pulmonary valve; RC, right cusp; AC, anterior cusp; LC, left cusp. 
Table 6 Aortic arch defects (Vascular rings) causing tracheoesophageal compression

\begin{tabular}{|c|c|c|}
\hline Aortic arch anomalies & Clinical picture & Surgical management \\
\hline Right aortic arch with left-sided ligamentum arteriosus & Wheezing; dysphagia & Interruption of ligamentum arteriosus \\
\hline $\begin{array}{l}\text { Right aortic arch with left-sided ligamentum arteriosus and } \\
\text { Kommerell diverticulum }\end{array}$ & Wheezing; dysphagia & $\begin{array}{l}\text { Resection of Kommerell diverticulum; } \\
\text { interruption of ligamentum arteriosus }\end{array}$ \\
\hline
\end{tabular}

Double aortic arch, right aortic arch with left ligamentum arteriosus and right aortic arch with left ligamentum arteriosus and Kommerell diverticulum are the most common forms of vascular ring sharing the anatomic site of tracheo-esophageal compression: The right side of tracheal carina at the origin of right main bronchus and the posterior wall of esophagus. The clinical picture of these lesions is dominated by wheezing and dysphagia. A much rarer form of vascular ring is represented by pulmonary artery sling, where the left pulmonary artery takes its origin from the right one and courses leftward passing between trachea and esophagus. In this situation the tracheal compression is posterior. The distinctive hallmark, only found in this type of vascular ring, is the anterior compression of the esophagus.

definition and perioperative management of vascular rings is therefore intuitive (78-80) (Table 6).

\section{Structural interventional cardiology}

In the context of structural heart intervention there are numerous interventions which benefit from CCTA, which include transcatheter aortic valve implantation (TAVI), percutaneous interventions of the mitral, tricuspid valves, left atrial appendage occlusion (LAAO) and the interventional closure of paravalvular leaks related to prosthetic valve replacement $(41,81-88)$ (Tables 7,8$)$.

The evaluation of aortic root and aortic annulus dimensions, assessment of aortic valve structure and calcifications, distance between the aortic annulus and the coronary ostia, determination of fluoroscopic viewing angles as well as analysis of the vascular access route should always be included $(41,45,81-91)$.

The technique of transcatheter MV implantation (TMVI) has been further developed $(83,86)$. The preprocedural $\mathrm{CT}$ imaging in this contest must address anatomical assessment and measurement of the mitral valvular and subvalvular apparatus, assessment of annular calcification, prediction of possible left ventricular outflow tract (LVOT) obstruction, investigation of mitral regurgitation etiology and prediction of an orthogonal fluoroscopic angulation. Moreover, a CCTA imaging is necessarily to define the anatomic relationship of the coronary sinus, the left circumflex CA, and the mitral annulus prior to a mitral annuloplasty procedure (85) (Tables 9,10). For the post-procedural assessment of valvular and paravalvular complications, CCTA allows the visualization of the possible incomplete opening or closing of prosthetic mechanical cusps and the complication of most biological valves due to pannus ingrowth or thrombosis formation (92). However, TAVI candidates, may be ineligible to CT in up to $20 \%$ of cases due to coexisting borderline renal function. In such cases, CMR may be a valid alternative to CT (32). For ring annuloplasty, a nonplanar design of the surgical ring is suggested to improve ventricular function (93). The ring is C-shaped, and its gap is placed at the site of the septal leaflet to prevent complication to the conduction system. When transcatheter valve implantation into a surgically placed ring or prosthesis is considered (either in aortic, tricuspid or mitral position), the valve size can be selected based on the sewing ring's nominal size or on the measurements of the reconstructed CCTA views of the valve prosthesis or ring (93). The LAAO need a correct sizing for a successful device deployment (94). LAA morphology, ostial diameters and depth, the landing-zone as well as the presence of thrombi and the assessment of surrounding structures (pulmonary veins, mitral annulus, left circumflex artery) (95) should be managed in a multimodality fashion $(96,97)$. CCTA can provide detailed information on the exact location and morphological characteristics of paravalvular leaks as common complication of surgical and interventional $(98,99)$, including size, course and shape as well as the presence of multiple communications (100-104).

\section{Congenital interventional cardiology}

Interventional cardiology is nowadays a feasible and costeffective alternative to surgery for palliation or repair of 
Table 7 Imaging methods for the evaluation of patients' anatomical suitability for TAVI according to current practice

\begin{tabular}{|c|c|c|c|c|}
\hline & TTE/TOE & CCTA & CMR & Angiography \\
\hline LV function & +++ & $++^{*}$ & ++ & - \\
\hline LV septal thickness & ++ & $++^{*}$ & +++ & - \\
\hline Concomitant valvular disease & +++ & + & +++ & - \\
\hline AV anatomy & ++ & +++ & ++ & - \\
\hline AV calcification & ++ & +++ & - & ++ \\
\hline Aortic root measurements & ++ & +++ & +++ & ++ \\
\hline AV annulus - coronary arteries distance & \pm & +++ & +++ & \pm \\
\hline Peripheral arteries anatomy & - & +++ & ++ & ++ \\
\hline Peripheral arteries calcification & - & +++ & - & + \\
\hline
\end{tabular}

+++ , most frequently used; + +, less frequently used; +, least used; -, unsuitable; *, related to the retrospective multiphasic acquisition. AS, aortic stenosis; LV, left ventricular; AV, aortic annulus; TTE, transthoracic echocardiography; TOE, transoesophageal echocardiography; CCTA, cardiac computed tomography angiography; CMR, cardiac magnetic resonance.

Table 8 Role of CCTA in transcatheter tricuspid valve interventions

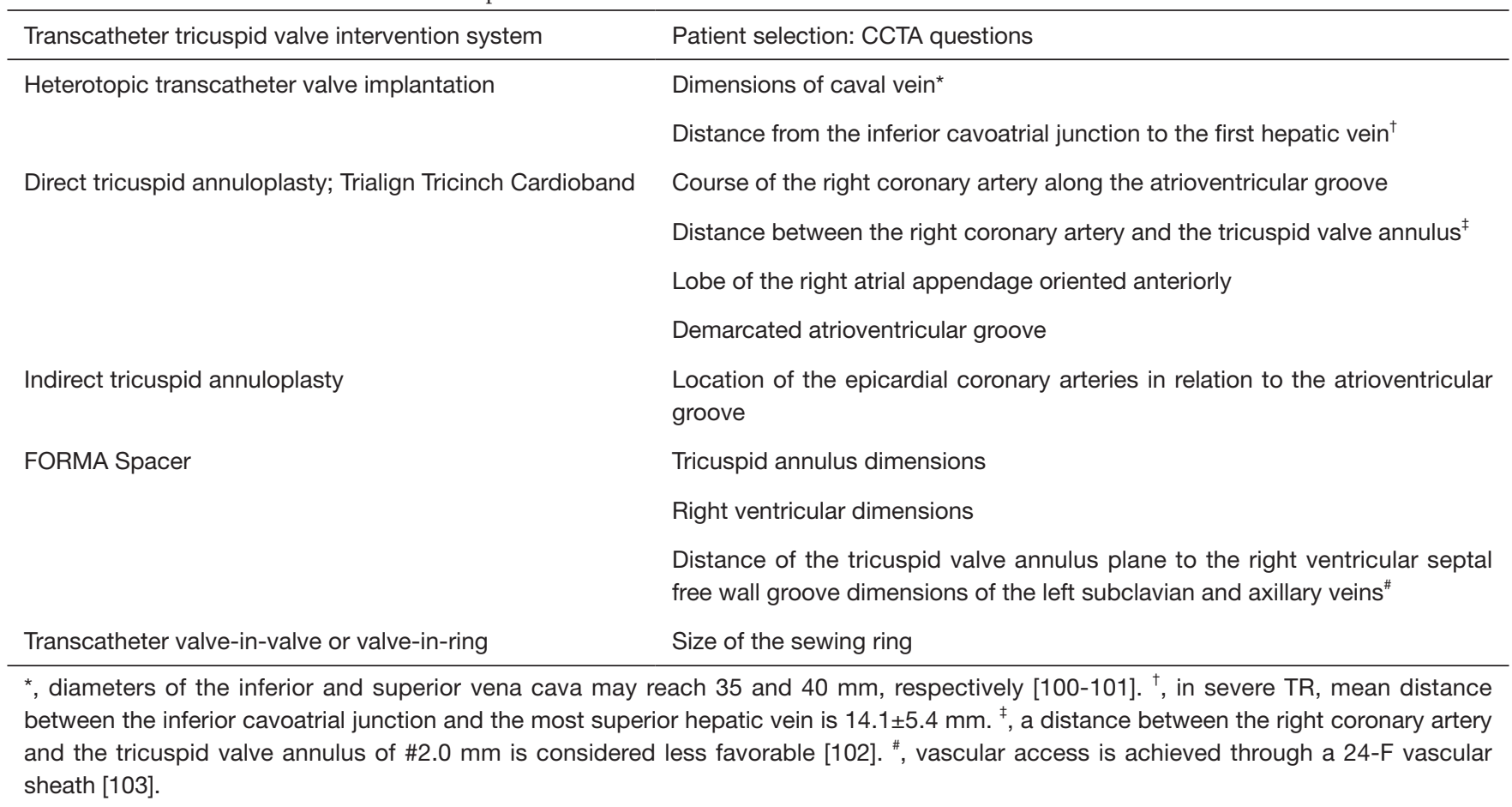


Table 9 Imaging role for mitral valve intervention

\begin{tabular}{|c|c|c|c|c|}
\hline Main tasks & TTE & 2D/3D TEE & CCTA & MRI \\
\hline Grading of MR/MS severity & $x$ & & & \\
\hline Associated valve/heart disease & $x$ & & & \\
\hline LV/LA function & $x$ & & $\mathrm{x}^{*}$ & \\
\hline Detailed assessment of MV pathology & & $x$ & & \\
\hline Re-confirmation MS/MR severity & & $x$ & & \\
\hline Exclusion of thrombi/infective endocarditis/pericardial effusion & & $x$ & $x$ & \\
\hline Exclusion of specific $\mathrm{Cl}$ for the planned procedure & & $x$ & $x$ & \\
\hline Localization/extent of calcification of structures of MV apparatus & & & $x$ & \\
\hline $\begin{array}{l}\text { Anatomical relationship of target lesions to surrounding cardiac/ } \\
\text { extracardiac structures }\end{array}$ & & & $x$ & \\
\hline Evaluation of chamber volumes and ejection fraction & & & $x^{*}$ & $x$ \\
\hline Regurgitant volumes & & & & $x$ \\
\hline Regional and global myocardial function & & & $x^{*}$ & $x$ \\
\hline
\end{tabular}

*, end-systolic and end-diastolic acquisition. $x$ indicates the capability of the imaging modality. MV, mitral valve; TTE, transthoracic echocardiography; TEE, transesophageal echocardiography; CCTA, cardiac computed tomography angiography; MRI, magnetic resonance imaging; MR, mitral regurgitation; MS, mitral stenosis; LV, left ventricular; LA, left atrial.

Table 10 Specific role of CCTA in various mitral valve pathologies and interventions

\begin{tabular}{lcccc}
\hline & $\begin{array}{c}\text { TMVR procedure } \\
\text { in MS }\end{array}$ & $\begin{array}{c}\text { TMVR procedure } \\
\text { in primary MR }\end{array}$ & $\begin{array}{c}\text { Direct/indirect } \\
\text { annuloplasty is } \\
\text { secondary MR }\end{array}$ & $\begin{array}{c}\text { Failed } \\
\text { bioprosthesis/ } \\
\text { annuloplasty }\end{array}$ \\
\hline $\begin{array}{l}\text { Annular dimensions } \\
\begin{array}{l}\text { Localization/extent of calcification } \\
\text { of structures of MV apparatus }\end{array}\end{array}$ & $\mathrm{x}$ & $\mathrm{x}$ & $\mathrm{x}$ & $\begin{array}{c}\mathrm{x} \text {; localization, number, size, } \\
\text { orientation of the leak(s) to } \\
\text { the sewing ring/prosthesis }\end{array}$ \\
$\begin{array}{l}\text { Anatomical relationship target } \\
\text { lesions to surrounding structures }\end{array}$ & $\begin{array}{c}\mathrm{x} \text {; aorto-mitral } \\
\text { angle }\end{array}$ & $\begin{array}{c}\mathrm{x} \text {; aorto-mitral } \\
\text { angle }\end{array}$ & $\begin{array}{c}\mathrm{x} \text {; relationship } \\
\text { annulus-LCx and CS }\end{array}$ & $\mathrm{x}$ transapical puncture site \\
\hline
\end{tabular}

2D, 2-dimensional; 3D, 3-dimensional; Cl, contraindication; CS, coronary sinus; CT, computed tomography; LA, left atrial; LCx, left circumflex coronary artery; LV, left ventricular; MR, mitral regurgitation; MRI, magnetic resonance imaging; MS, mitral stenosis; MV, mitral valve; PVML, paravalvular mitral leak; TEE, transesophageal echocardiography; TMVR, transcatheter mitral valve replacement; TTE, transthoracic echocardiography. $\mathrm{x}$, leading role of CCTA. 
CHD, with the aim of decreasing hospitalization length as well as morbidity and mortality rate (105). However, this approach is critically dependent on cardiovascular imaging either before or after any procedure. In this setting, CCTA may be crucial to image detailed morphologic features of complex cardiac malformations (106), thus limiting the need for sedation/general anaesthesia. 3D printed models manufacturing reproducing anatomical details, allows virtual and physical manipulation in planning percutaneous intervention (107). Ionizing radiations exposure, which is a concern in younger children and women, is nowadays limited by reduction dose protocols (108). 3D-virtual imaging and element modelling of intra- and extra-cardiac anatomy, based on CCTA images, may be particularly useful in finely diagnosing and planning complex transcatheter interventions (109). It may be particularly useful to plan the best route to access the cardiac cavity in certain interventional procedures, as well as in testing ex-vivo potentially useful devices. In fact, in patients submitted to multiple cardiac surgeries, the native anatomy is completely altered by fibrous changes as well as compensatory mechanisms due to pressure- or volume-overload conditions. A paradigmatic example is trans-catheter interventional treatment of aortic coarctation with balloon angioplasty stenting, that is nowadays considered as the first-line therapy in complex native or post-surgical settings (110-112). CCTA is also the first-line imaging modality in the evaluation of complex arch anomalies, like vascular rings and slings (113), to diagnose the type of anomaly and the relationship between the trachea and oesophagus, as well as major aortopulmonary collaterals (MAPCAs) in complex forms of tetralogy of Fallot (114).

CCTA details of coronary ostia, take-off from the aortic root and major branch peripheral distribution, mainly in terms of interarterial course of proximal segments or intramyocardial course of distal branches (115) are of paramount importance in the case of congenital cardiac malformations as well as in the evaluation of post-surgical changes following coronary reimplantation. This is also essential in planning a trans-catheter pulmonary valve implantation since the proximity of major CA branches to the right ventricular outflow tract may preclude the valve deployment due to the risk for coronary compression (116).

\section{Future perspectives: dual- and multi-energy CT}

During recent years, rapid advances in CT hardware and software technology of newer generation scanners have occurred, including dual-energy CT (DECT) and multi-energy CT imaging (117-119). DECT systems enable material differentiation by evaluating attenuation characteristics at two different photon energy levels. Several studies have demonstrated that DECT may enhance the diagnostic performance of CT in myocardial perfusion and scar imaging by improving iodine contrast-to-noise ratio (CNR) (119). DECT allows accurate quantification of Iodine contrast distribution within the myocardium, which is directly related to myocardial blood flow, thus being useful for different cardiac ECG-gated synchronization between normal, ischemic and necrotic myocardium (120). Color-coded iodine overlying images (Iodine maps) can be also generated by the dual-energy dataset, providing a measure of per-voxel iodine myocardial concentration expressed in $\mathrm{mg} / \mathrm{ml}$, which improves accuracy when compared to standard visual analysis. Moreover, DECT acquisition may improve limitations of single-energy CT such as beam hardening and blooming artifacts, without increasing radiation dose $(118,119)$.

Most recently, new energy-sensitive photon counting detectors (PCDs) have been developed which directly count the number of incident photons and measure their photon energies separately. Multi-energy CT with PCDs may provide more spectral information than DECT systems, but they are the subject of ongoing research and are not yet commercially available (61). A recent preclinical experimental model has demonstrated the feasibility and accuracy of PCDs compared to MRI and histology as the reference standard for quantitative separation of blood pool, scar, and remote myocardium using a simultaneous multicontrast agent protocol (121).

\section{Conclusions}

Normal cardiac CT anatomy, pathological changes, preand postoperative cardiac CT aspects are essential in the era of digital quantitative advanced bioimaging.

The structural development of CT scanners nowadays allows the virtual measurement of anatomical structures complying with heart rate with sub millimetric precision permitting to follow the pathology temporal evolution. CCTA asserts itself as a gold standard method for the anatomical evaluation of the heart and permits to evaluate, verify, measure and characterize structural pathological alterations of both congenital and acquired degenerative. 
The multi-energy CT scanners will increasingly provide not only static and dynamic anatomical features, but also metabolic alterations.

\section{Acknowledgments}

Funding: None.

\section{Footnote}

Provenance and Peer Review: This article was commissioned by the editorial office, Cardiovascular Diagnosis and Therapy for the series "Impact of Cardiac CT in Clinical Practice". The article has undergone external peer review.

Reporting Checklist: The authors have completed the Narrative Review reporting checklist. Available at http:// dx.doi.org/10.21037/cdt-20-530

Conflicts of Interest: All authors have completed the ICMJE uniform disclosure form (available at http://dx.doi. org/10.21037/cdt-20-530). The series "Clinical Impact of Cardiac CT in Clinical Practice" was commissioned by the editorial office without any funding or sponsorship. FC served as the unpaid Guest Editor of the series and serves as an unpaid editorial board member of Cardiovascular Diagnosis and Therapy from Jul 2019 to Jun 2021. All authors declares that they have never received payment or services from a third party for any aspect of the submitted work, that they didn't have financial relationships with entities during the 36 months prior to publication, they do not have any patents, whether planned, pending or issued, broadly relevant to the work, that no other relationships/conditions/ circumstances are present a potential conflict of interest are present.

Ethical Statement: The authors are accountable for all aspects of the work in ensuring that questions related to the accuracy or integrity of any part of the work are appropriately investigated and resolved.

Open Access Statement: This is an Open Access article distributed in accordance with the Creative Commons Attribution-NonCommercial-NoDerivs 4.0 International License (CC BY-NC-ND 4.0), which permits the noncommercial replication and distribution of the article with the strict proviso that no changes or edits are made and the original work is properly cited (including links to both the formal publication through the relevant DOI and the license). See: https://creativecommons.org/licenses/by-nc-nd/4.0/.

\section{References}

1. Shoja MM, Agutter PS, Loukas M, et al. Leonardo da Vinci's studies of the heart. Int J Cardiol 2013;167:1126-33.

2. Zampieri F, Zanatta A, Basso C, et al. Cardiovascular medicine in Morgagni's De sedibus: dawn of cardiovascular pathology. Cardiovasc Pathol 2016;25:443-52.

3. Hendel RC, Patel MR, Kramer CM, et al. ACCF/ ACR/SCCT/SCMR/ASNC/NASCI/SCAI/SIR 2006 appropriateness criteria for cardiac computed tomography and cardiac magnetic resonance imaging: a report of the American College of Cardiology Foundation Quality Strategic Directions Committee Appropriateness Criteria Working Group, American College of Radiology, Society of Cardiovascular Computed Tomography, Society for Cardiovascular Magnetic Resonance, American Society of Nuclear Cardiology, North American Society for Cardiac Imaging, Society for Cardiovascular Angiography and Interventions, and Society of Interventional Radiology. J Am Coll Cardiol 2006;48:1475-97.

4. Clayton B, Roobottom C, Morgan-Hughes G. Assessment of the myocardium with cardiac computed tomography. Eur Heart J Cardiovasc Imaging 2014;15:603-9.

5. YamamuroM, Tadamura E, Kubo S, et al. Cardiac functional analysis with multi-detector row $\mathrm{CT}$ and segmental reconstruction algorithm: comparison with echocardiography, SPECT, and MR imaging. Radiology 2005;234:381-90.

6. Burianová L, Riedlbauchova L, Lefflerova K, et al. Assessment of left ventricular function in non-dilated and dilated hearts: comparison of contrast-enhanced 2-dimensional echocardiography with multi-detector row CT angiography. Acta Cardiol 2009;64:787-94.

7. Kang EJ, Lee KN, Choi WJ, et al. Left Ventricular Functional Parameters and Geometric Patterns in Korean Adults on Coronary CT Angiography with a 320-DetectorRow CT Scanner. Korean J Radiol 2017;18:664-73.

8. Palumbo A, Maffei E, Martini C, et al. Functional parameters of the left ventricle: comparison of cardiac MRI and cardiac CT in a large population. Radiol Med 2010;115:702-13.

9. de Graaf FR, Schuijf JD, van Velzen JE, et al. Assessment of global left ventricular function and volumes with 320-row multidetector computed tomography: A comparison with 2D-Echocardiography. 
J Nucl Cardiol 2010;17:225-31.

10. Kara B, Nayman A, Guler I, et al. Quantitative Assessment of Left Ventricular Function and Myocardial Mass: A Comparison of Coronary CT Angiography with Cardiac MRI and Echocardiography. Pol J Radiol 2016;81:95-102.

11. Stolzmann P, Scheffel H, Leschka S, et al. Reference values for quantitative left ventricular and left atrial measurements in cardiac computed tomography. Eur Radiol 2008;18:1625-34.

12. Maffei E, Messalli G, Martini C, et al. Left and right ventricle assessment with Cardiac CT: validation study vs. Cardiac MR. Eur Radiol 2012;22:1041-9.

13. Kawel-Boehm N, Maceira A, Valsangiacomo-Buechel ER, et al. Normal values for cardiovascular magnetic resonance in adults and children. J Cardiovasc Magn Reson 2015;17:29.

14. Cademartiri F, Seitun S, Clemente A, et al. Myocardial blood flow quantification for evaluation of coronary artery disease by computed tomography. Cardiovasc Diagn Ther 2017;7:129-50.

15. Lardo AC, Cordeiro MA, Silva C, et al. Contrastenhanced multidetector computed tomography viability imaging after myocardial infarction: characterization of myocyte death, microvascular obstruction, and chronic scar. Circulation 2006;113:394-404.

16. Gerber BL, Belge B, Legros GJ, et al. Characterization of acute and chronic myocardial infarcts by multidetector computed tomography: comparison with contrast-enhanced magnetic resonance. Circulation 2006;113:823-33.

17. Marcus FI, McKenna WJ, Sherrill D, et al. Diagnosis of arrhythmogenic right ventricular cardiomyopathy/ dysplasia: proposed modification of the task force criteria. Circulation 2010;121:1533-41.

18. Cannavale G, Francone M, Galea N, et al. Fatty Images of the Heart: Spectrum of Normal and Pathological Findings by Computed Tomography and Cardiac Magnetic Resonance Imaging. Biomed Res Int 2018;2018:5610347.

19. Mastrodicasa D, Albrecht MH, Schoepf UJ, et al. Artificial intelligence machine learning-based coronary CT fractional flow reserve (CT-FFRML): Impact of iterative and filtered back projection reconstruction techniques. J Cardiovasc Comput Tomogr 2019;13:331-5.

20. Makkuni P, Kotler MN, Figueredo VM. Diverticular and aneurysmal structures of the left ventricle in adults: report of a case within the context of a literature review. Tex Heart Inst J 2010;37:699-705

21. Axsom K, Lin F, Weinsaft JW, et al. Evaluation of myocarditis with delayed-enhancement computed tomography. J Cardiovasc Comput Tomogr 2009;3:409-11.

22. Ricci F, Mantini C, Grigoratos C, et al. The Multimodality Cardiac Imaging Approach to Cardiac Sarcoidosis. Curr Med Imaging Rev 2019;15:10-20.

23. Rizvi A, Deaño RC, Bachman DP, et al. Analysis of ventricular function by CT. J Cardiovasc Comput Tomogr 2015;9:1-12.

24. Taylor AJ, Cerqueira M, Hodgson JM, et al. ACCF/ SCCT/ACR/AHA/ASE/ASNC/NASCI/SCAI/SCMR 2010 appropriate use criteria for cardiac computed tomography. J Am Coll Cardiol 2010;56:1864-94.

25. Gandjbakhch E, Redheuil A, Pousset F, et al. Clinical Diagnosis, Imaging, and Genetics of Arrhythmogenic Right Ventricular Cardiomyopathy/Dysplasia: JACC State-of-the-Art Review. J Am Coll Cardiol 2018;72:784-804.

26. Piazza N, de Jaegere P, Schultz C, et al. Anatomy of the aortic valvar complex and its implications for transcatheter implantation of the aortic valve. Circ Cardiovasc Interv 2008;1:74-81.

27. Buchner S, Kobuch R, Luchner A, et al. Diagnosis of unicommissural unicuspid aortic valve stenosis by different imaging modalities. J Cardiovasc Med (Hagerstown) 2011;12:347-8.

28. Liu T, Xie M, Lv Q, et al. Bicuspid aortic valve: An update in morphology, genetics, biomarker, complications, imaging diagnosis and treatment. Front Physiol 2019;9:1921.

29. Sievers HH, Schmidtke C. A classification system for the bicuspid aortic valve from 304 surgical specimens. J Thorac Cardiovasc Surg 2007;133:1226-33.

30. Khan SK, Tamin SS, Araoz PA. Quadricuspid aortic valve by cardiac magnetic resonance imaging: a case report and review of the literature. J Comput Assist Tomogr 2011;35:637-41.

31. Tutarel $O$. The quadricuspid aortic valve: a comprehensive review. J Heart Valve Dis 2004;13:534-7.

32. Mantini C, Di Giammarco G, Pizzicannella J, et al. Grading of aortic stenosis severity: a head-to-head comparison between cardiac magnetic resonance imaging and echocardiography. Radiol Med 2018;123:643-54.

33. McCarthy KP, Ring L, Rana BS. Anatomy of the mitral valve: understanding the mitral valve complex in mitral regurgitation. Eur J Echocardiogr 2010;11:i3-i9.

34. Harb SC, Griffin BP. Mitral Valve Disease: a Comprehensive Review. Curr Cardiol Rep 2017;19:73.

35. Gunnal SA, Wabale RN, Farooqui MS. Morphological 
variations of papillary muscles in the mitral valve complex in human cadaveric hearts. Singapore Med J 2013;54:44-8.

36. Axel L. Papillary muscles do not attach directly to the solid heart wall. Circulation 2004;109:3145-8.

37. Khan MS, Biederman R. Dynamic cardiac anatomy: the "cypress tree" papillary muscle root. J Cardiovasc Thorac Res 2018;10:138-43.

38. Morris MF, Maleszewski JJ, Suri RM, et al. CT and MR imaging of the mitral valve: radiologic-pathologic correlation. Radiographics 2010;30:1603-20.

39. Lembcke A, Durmus T, Westermann Y, et al. Assessment of mitral valve stenosis by helical MDCT: comparison with transthoracic doppler echocardiography and cardiac catheterization. AJR Am J Roentgenol 2011;197:614-22.

40. Weir-McCall JR, Blanke P, Naoum C, et al. Mitral Valve Imaging with CT: Relationship with Transcatheter Mitral Valve Interventions. Radiology 2018;288:638-55.

41. Baumgartner H, Falk V, Bax J, et al. 2017 ESC/EACTS Guidelines for the management of valvular heart disease. Eur Heart J 2017;38:2739-91.

42. Naoum C, Blanke P, Cavalcante JL, et al. Cardiac Computed Tomography and Magnetic Resonance Imaging in the Evaluation of Mitral and Tricuspid Valve Disease: Implications for Transcatheter Interventions. Circ Cardiovasc Imaging 2017;10:e005331.

43. van Rosendael PJ, Kamperidis V, Kong WK, et al. Computed tomography for planning transcatheter tricuspid valve therapy. Eur Heart J 2017;38:665-74.

44. Saremi F, Sánchez-Quintana D, Mori S, et al. Fibrous Skeleton of the Heart: Anatomic Overview and Evaluation of Pathologic Conditions with CT and MR Imaging. Radiographics 2017;37:1330-51.

45. Fukuda S, Saracino G, Matsumura Y, et al. Threedimensional geometry of the tricuspid annulus in healthy subjects and in patients with functional tricuspid regurgitation: a real-time, 3-dimensional echocardiographic study. Circulation 2006;114:I492-I498.

46. Tei C, Pilgrim JP, Shah PM, et al. The tricuspid valve annulus: study of size and motion in normal subjects and in patients with tricuspid regurgitation. Circulation 1982;66:665-71.

47. Ton-Nu TT, Levine RA, Handschumacher MD, et al. Geometric determinants of functional tricuspid regurgitation: insights from 3-dimensional echocardiography. Circulation 2006;114:143-9.

48. Bogaert J, Francone M. Pericardial disease: value of CT and MR imaging. Radiology 2013;267:340-56.

49. Calabri G, Clemente A, Santoro G. Repeat percutaneous recanalizations of a discontinuous pulmonary artery: A very "lucky" vessel. Ann Pediatr Cardiol 2020;13:163-6.

50. Han BK, Rigsby CK, Leipsic J, et al. Computed tomography imaging in patients with congenital heart disease, part 2: technical recommendations. An expert consensus document of the Society of Cardiovascular Computed Tomography (SCCT): endorsed by the Society of Pediatric Radiology (SPR) and the North American Society of Cardiac Imaging (NASCI). J Cardiovasc Comput Tomogr 2015;9:493-513.

51. Truong QA, Massaro JM, Rogers IS, et al. Reference values for normal pulmonary artery dimensions by noncontrast cardiac computed tomography: the Framingham Heart Study. Circ Cardiovasc Imaging 2012;5:147-54.

52. Moore AJE, Wachsmann J, Chamarthy MR, et al. Imaging of acute pulmonary embolism: an update. Cardiovasc Diagn Ther 2018;8:225-43.

53. Bannister LH, Berry MM, Collins P, al. e. Gray's Anatomy - Section 10: Cardiovascular System, Pericardium pag 1471-2. 38th edition ed. New York Edinburgh: Churchill Livingstone; 1995.

54. Kligerman S. Imaging of pericardial disease. Radiol Clin N Am 2019;57:179-99.

55. O'Leary SM, Williams PL, Williams MP, et al. Imaging the pericardium: appearances on ECG-gated 64-detector row cardiac computed tomography. Br J Radiol 2010;83:194-205.

56. Restrepo CS, Lemos DF, Lemos JA, et al. Imaging findings in cardiac tamponade with emphasis on CT. Radiographics 2007;27:1595-610.

57. Hammer MM, Raptis CA, Javidan-Nejad C, et al. Accuracy of computed tomography findings in acute pericarditis. Acta Radiol 2014;55:1197-202.

58. Waller BF, Taliercio CP, Howard J, et al. Morphologic aspects of pericardial heart disease: part I. Clin Cardiol 1992;15:203-9.

59. Blomström Lundqvist C, Auricchio A, Brugada J, et al. The use of imaging for electrophysiological and devices procedures: a report from the first European Heart Rhythm Association Policy Conference, jointly organized with the European Association of Cardiovascular Imaging (EACVI), the Council of Cardiovascular Imaging and the European Society of Cardiac Radiology. Europace 2013;15:927-36.

60. Donal E, Lip GYH, Galderisi M, et al. EACVI/EHRA Expert Consensus Document on the role of multimodality imaging for the evaluation of patients with atrial fibrillation. Eur Heart J Cardiovasc Imaging 
2016;17:355-83.

61. Sanchis L, Prat S, Sitges M. Cardiovascular Imaging in the Electrophysiology Laboratory. Rev Esp Cardiol 2016;69:595-605.

62. Kuchynka P, Podzimkova J, Masek M, et al. The Role of Magnetic Resonance Imaging and Cardiac Computed Tomography in the Assessment of Left Atrial Anatomy, Size, and Function. Rev Esp Cardiol 2016;69:595-605.

63. Clemente A, Traghella I, Mazzone A, et al. Vascular and valvular calcification biomarkers. Adv Clin Chem 2020;95:73-103.

64. Beller GA, Bonow RO, Fuster V. ACCF 2008 recommendations for training in adult cardiovascular medicine core cardiology training (COCATS 3) (revision of the 2002 COCATS Training Statement). J Am Coll Cardiol 2008;51:335-8.

65. Leipsic J, Abbara S, Achenbach S, et al. SCCT guidelines for the interpretation and reporting of coronary CT angiography: A report of the Society of Cardiovascular Computed Tomography guidelines committee. J Cardiovasc Comput Tomogr 2014;8:342-58.

66. Maffei E, Arcadi T, Zuccarelli A, et al. The impact of training on diagnostic accuracy with computed tomography coronary angiography. J Cardiovasc Med (Hagerstown) 2013;14:719-25.

67. Austen WG, Edwards JE, Frye RL, et al. A reporting system on patients evaluated for coronary artery disease. Report of the Ad Hoc Committee for Grading of Coronary Artery Disease, Council on Cardiovascular Surgery, American Heart Association. Circulation 1975;51:5-40.

68. Angelini P, Villason S, Chan AV Jr, et al. Normal and anomalous coronary arteries in humans. In: Angelini P. editor. Coronary artery anomalies: a comprehensive approach. Philadelphia: Lippincott Williams \& Wilkins; 1999:27-150.

69. Angelini P. Coronary artery anomalies--current clinical issues: definitions, classification, incidence, clinical relevance, and treatment guidelines. Tex Heart Inst J 2002;29:271-8.

70. Pérez-Pomares JM, de la Pompa JL, Franco D, et al. Congenital coronary artery anomalies: a bridge from embryology to anatomy and pathophysiology — a position statement of the development, anatomy, and pathology ESC Working Group. Cardiovasc Res 2016;109:204-16

71. Ou P, Celermajer DS, Calcagni G, et al. Three-dimensional CT-scanning: A new diagnostic modality in congenital heart disease. Heart 2007;93:908-13.

72. Imran Hamid U, Digney R, Soo L, et al. Incidence and outcome of re-entry injury in redo cardiac surgery: Benefits of preoperative planning. Eur J Cardiothorac Surg 2015;47:819-23.

73. Shi G, Zhu Z, Chen J, et al. Total Anomalous Pulmonary Venous Connection: The Current Management Strategies in a Pediatric Cohort of 768 Patients. Circulation 2017;135:48-58.

74. Mery CM, De Leòn LE, Molossi S, et al. Outcomes of surgical intervention for anomalous aortic origin of a coronary artery: A large contemporary prospective cohort study. J Thorac cardiovasc Surg 2018;155:305-319.e4.

75. Gosnell J, Pietila T, Samuel BP, et al. Integration of a computed tomography and three-dimensional echocardiography for hybrid three- dimensional printing in congenital heart disease. J Digit Imaging 2016;29:665-9.

76. Hongu H, Yamagishi M, Myazaki T, et al. Late results of Half-Turned truncal switch operation for transposition of the great arteries. Ann Thorac Surg 2018;106:1421-8.

77. Yacoub MH, Radley-Smith R. Anatomy of the coronary arteries in transposition of the great arteries and methods for their transfer in anatomical correction. Thorax 1978;33:418-24.

78. Hazekamp MG, Nevvazhay T, Sojak V. Nikaidoh vs Réparation à l'etage ventriculaire vs Rastelli. Semin Thorac Cardiovasc Surg Pediatr Card Surg Annu 2018;21:58-63.

79. Backer CL, Hilmann N, Mavroudis C, et al. Resection of Kommerell's diverticulum and left subclavian artery transfer For recurrent symptoms after vascular ring division. Eur J Cardiothorac Surg 2002;22:64-9.

80. Backer CL, Mongé MC, Popescu AR, et al. Vascular rings. Semin Pediatr Surg 2016;25:165-75.

81. Ippolito D, Riva L, Talei Franzesi C, et al. Computed Tomography Angiography Combined With KnowledgeBased Iterative Algorithm for Transcatheter Aortic Valve Implantation Planning: Image Quality and Radiation Dose Exposure With Low-kV and Low-Contrast-Medium Protocol. J Comput Assist Tomogr 2020;44:13-9.

82. Achenbach S, Delgado V, Hausleiter J, et al. SCCT expert consensus document on computed tomography imaging before transcatheter aortic valve implantation (TAVI)/transcatheter aortic valve replacement (TAVR). J Cardiovasc Comput Tomogr 2012;6:366-80.

83. Bapat V, Buellesfeld L, Peterson MD, et al. Transcatheter mitral valve implantation (TMVI) using the Edwards fortis device. EuroIntervention 2014;10 Suppl U:U120-U128.

84. Sondergaard L, Brooks M, Ihlemann N, Vet al. Transcatheter mitral valve implantation via transapical approach: an early experience. Eur J Cardiothorac Surg 
2015;48:873-8 discussion 877-8.

85. Wunderlich NC, Beigel R, Ho SY, et al. Imaging for Mitral Interventions: Methods and Efficacy. JACC Cardiovascular Imaging 2018;11:872-901.

86. Choure AJ, Garcia MJ, Hesse B, et al. In vivo analysis of the anatomical relationship of coronary sinus to mitral annulus and left circumflex coronary artery using cardiac multidetector computed tomography: implications for percutaneous coronary sinus mitral annuloplasty. J Am Coll Cardiol 2006;48:1938-45.

87. van Rosendael PJ, Joyce E, Katsanos S, et al. Tricuspid valve remodeling in functional tricuspid regurgitation: multidetector row computed tomography insights. Eur Heart J Cardiovasc Imaging 2016;17:96-105.

88. Delgado V, Ng AC, van de Veire NR, et al. Transcatheter aortic valve implantation: role of multi-detector row computed tomography to evaluate prosthesis positioning and deployment in relation to valve function. Eur Heart J 2010;31:1114-23.

89. Yucel-Finn A, Nicol E, Leipsic JA, et al. CT in planning transcatheter aortic valve implantation procedures and risk assessment. Clin Radiol 2019. [Epub ahead of print].

90. Zamorano JL, Gonçalves A, Lang R. Imaging to select and guide transcatheter aortic valve implantation. Eur Heart J 2014;35:1578-87.

91. Pham N, Zaitoun H, Mohammed TL, et al. Complications of aortic valve surgery: manifestations at CT and MR imaging. Radiographics 2012;32:1873-92.

92. Ravani M, Koni E, Al Jabri A, et al. Transcatheter tricuspid valve-in-valve replacement in patients with large degenerated bioprostheses: Two case reports treated with Sapien 3 device using the new Ultra delivery System. Cardiovasc Revasc Med 2020. [Epub ahead of print].

93. Rogers JH, Bolling SF. Valve repair for functional tricuspid valve regurgitation: anatomical and surgical considerations. Semin Thorac Cardiovasc Surg 2010;22:84-9.

94. Clemente A, Avogliero F, Berti S, et al. Multimodality imaging in preoperative assessment of left atrial appendage transcatheter occlusion with the Amplatzer Cardiac Plug. Eur Heart J Cardiovasc Imaging 2015;16:1276-87.

95. Korsholm K, Jensen JM, Nørgaard BL, et al. Detection of Device-Related Thrombosis Following Left Atrial Appendage Occlusion: A Comparison Between Cardiac Computed Tomography and Transesophageal Echocardiography. Circ Cardiovasc Interv 2019;12:e008112.

96. Korsholm K, Berti S, Iriart X, et al. Expert Recommendations on Cardiac Computed Tomography for
Planning Transcatheter Left Atrial Appendage Occlusion. JACC Cardiovasc Interv 2020;13:277-92.

97. Fanni, BM, Capellini K, Di Leonardo M, et al. Correlation between LAA Morphological Features and Computational Fluid Dynamics Analysis for Non-Valvular Atrial Fibrillation Patients. Appl Sci 2020;10:1448.

98. Ionescu A, Fraser AG, Butchart EG. Prevalence and clinical significance of incidental paraprosthetic valvar regurgitation: a prospective study using transoesophageal echocardiography. Heart 2003;89:1316-21.

99. Lesser JR, Han BK, Newell M, et al. Use of cardiac CT angiography to assist in the diagnosis and treatment of aortic prosthetic paravalvular leak: a practical guide. J Cardiovasc Comput Tomogr 2015;9:159-64.

100.Laule M, Stangl V, Sanad W, et al. Percutaneous transfemoral management of severe secondary tricuspid regurgitation with Edwards Sapien XT bioprosthesis: firstin-man experience. J Am Coll Cardiol 2013;61:1929-31.

101.O'Neill BP, Wheatley G, Bashir R, et al. Study design and rationale of the heterotopic implantation of the EdwardsSapien XT transcatheter valve in the inferior Vena cava for the treatment of severe tricuspid regurgitation (HOVER) trial. Catheter Cardiovasc Interv 2016;88:287-93.

102. Díez-Villanueva P, Gutierrez-Ibanes E, Cuerpo-Caballero GP, et al. Direct injury to right coronary artery in patients undergoing tricuspid annuloplasty. Ann Thorac Surg 2014;97:1300-5.

103. Schöfer J, Bijuklic K, Tiburtius C, et al. First-in-human transcatheter tricuspid valve repair in a patient with severely regurgitant tricuspid valve. J Am Coll Cardiol 2015;65:1190-5.

104.Hahn RT, Meduri CU, Davidson CJ, et al. Early feasibility study of a transcatheter tricuspid valve annuloplasty: SCOUT trial 30-day results. J Am Coll Cardiol 2017;69:1795-806.

105. Bentham JR, Thomson JDR. Current state of interventional cardiology in congenital heart disease. Arch Dis Child 2015;100:787-92.

106. Goitein O., Salem Y., Jacobson J, et al. The role of cardiac computed tomography in infants with congenital heart disease. Isr Med Assoc J 2014;16:147-52.

107. Kurenov SN, Ionita C, Sammons D, et al. Threedimensional printing to facilitate anatomic study, device development, simulation, and planning in thoracic surgery. J Thorac Cardiovasc Surg 2015;149:973-9.e1.

108. Maffei E, Martini C, De Crescenzo S, et al. Low dose CT of the heart: a quantum leap into a new era of cardiovascular imaging. Radiol Med 2010;115:1179-207. 
109.Harb SC, Rodriguez LL, Vukicevic M, et al. ThreeDimensional Printing Applications in Percutaneous Structural Heart Interventions. Circ Cardiovasc Imaging 2019;12:e009014.

110.Pedra CAC, Fontes VF, Esteves CA, et al. Stenting vs. balloon angioplasty for discrete unoperated coarctation of the aorta in adolescents and adults. Catheter Cardiovasc Interv 2005;64:495-506.

111. Raimondi F, Warin-Fresse K. Computed tomography imaging in children with congenital heart disease: Indications and radiation dose optimization. Arch Cardiovasc Dis 2016;109:150-7.

112. Karaosmanoglu AD, Khawaja RDA, Onur MR, et al. CT and MRI of aortic coarctation: pre- and postsurgical findings. AJR Am J Roentgenol 2015;204:W224-33.

113. Etesami M, Ashwath R, Kanne J, et al. Computed tomography in the evaluation of vascular rings and slings. Insights Imaging 2014;5:507-21.

114. Meinel FG, Huda W, Schoepf UJ, et al. Diagnostic accuracy of CT angiography in infants with tetralogy of Fallot with pulmonary atresia and major aortopulmonary collateral arteries. J Cardiovasc Comput Tomogr 2013;7:367-75.

115.Rajiah P, Saboo SS, Abbara S. Role of CT in Congenital
Heart Disease. Curr Treat Options Cardiovasc Med 2017;19:6.

116.Peng LF, McElhinney DB, Nugent AW, et al. Endovascular stenting of obstructed right ventricleto-pulmonary artery conduits: A 15-year experience. Circulation 2006;113:2598-605.

117.McCollough CH, Leng S, Yu L, et al. Dual- and MultiEnergy CT: Principles, Technical Approaches, and Clinical Applications. Radiology 2015;276:637-53.

118. Danad I, Fayad ZA, Willemink MJ, et al. New applications of cardiac computed tomography: dual-energy, spectral, and molecular CT imaging. JACC Cardiovasc Imaging 2015;8:710-23.

119.Danad I, Ó Hartaigh B, Min JK. Dual-energy computed tomography for detection of coronary artery disease. Expert Rev Cardiovasc Ther 2015;13:1345-56.

120.Pelgrim GJ, van Hamersvelt RW, Willemink MJ, et al. Accuracy of iodine quantification using dual energy CT in latest generation dual source and dual layer CT. Eur Radiol 2017;27:3904-12.

121. Symons R, Cork TE, Lakshmanan MN, et al. Dualcontrast agent photon-counting computed tomography of the heart: initial experience. Int J Cardiovasc Imaging 2017;33:1253-61.
Cite this article as: Clemente A, Seitun S, Mantini C, Gentile G, Federici D, Barison A, Rossi A, Cuman M, Pizzuto A, AitAli L, Bossone E, Cademartiri F, Chiappino D. Cardiac CT angiography: normal and pathological anatomical features-a narrative review. Cardiovasc Diagn Ther 2020;10(6):1918-1945. doi: $10.21037 /$ cdt-20-530 